\title{
Fraction of bolometric luminosity absorbed by dust in DustPedia galaxies ${ }^{\star}$
}

S. Bianchi ${ }^{1}$, P. De Vis ${ }^{2}$, S. Viaene ${ }^{3,4}$, A. Nersesian ${ }^{3,5,6}$, A. V. Mosenkov ${ }^{7,8}$, E. M. Xilouris ${ }^{5}$, M. Baes ${ }^{3}$, V. Casasola ${ }^{1}$, L. P. Cassara ${ }^{5,9}$, C. J. R. Clark ${ }^{10}$, J. I. Davies ${ }^{10}$, I. De Looze ${ }^{3,11}$, W. Dobbels ${ }^{3}$, M. Galametz ${ }^{12}$, F. Galliano ${ }^{12}$, A. P. Jones ${ }^{2}$, S. Lianou ${ }^{12}$, S. C. Madden ${ }^{12}$, and A. Trčka ${ }^{3}$

1 INAF - Osservatorio Astrofisico di Arcetri, Largo E. Fermi 5, 50125 Florence, Italy e-mail: sbianchi@arcetri.astro.it

2 Institut d'Astrophysique Spatiale, UMR 8617, CNRS, Univerité Paris Sud, Univerité Paris-Saclay, Univerité Paris Sud, Orsay 91405, France

3 Sterrenkundig Observatorium, Universiteit Gent, Krijgslaan 281 S9, 9000 Gent, Belgium

4 Centre for Astrophysics Research, University of Hertfordshire, College Lane, Hatfield AL10 9AB, UK

5 National Observatory of Athens, Institute for Astronomy, Astrophysics, Space Applications and Remote Sensing, Ioannou Metaxa and Vasileos Pavlou, 15236 Athens, Greece

${ }^{6}$ Department of Astrophysics, Astronomy \& Mechanics, Faculty of Physics, University of Athens, Panepistimiopolis, 15784 Zografos, Athens, Greece

7 Central Astronomical Observatory of RAS, Pulkovskoye Chaussee 65/1, 196140 St. Petersburg, Russia

8 St. Petersburg State University, Universitetskij Pr. 28, 198504 St. Petersburg, Stary Peterhof, Russia

9 INAF - Istituto di Astrofisica Spaziale e Fisica Cosmica Milano, Via Alfonso Corti 12, 20133 Milan, Italy

10 School of Physics and Astronomy, Cardiff University, The Parade, Cardiff CF24 3AA, UK

11 Department of Physics and Astronomy, University College London, Gower Street, London WC1E 6BT, UK

12 Laboratoire AIM, CEA/DSM - CNRS - Université Paris Diderot, IRFU/Service d'Astrophysique, CEA Saclay, 91191 Gif-sur- Yvette, France

Received 22 June 2018 / Accepted 1 October 2018

\begin{abstract}
Aims. We aim to study the fraction of stellar radiation absorbed by dust, $f_{\mathrm{abs}}$, in 814 galaxies of different morphological types. The targets constitute the vast majority (93\%) of the DustPedia sample, including almost all large (optical diameter larger than $1^{\prime}$ ), nearby $\left(v \leq 3000 \mathrm{~km} \mathrm{~s}^{-1}\right)$ galaxies observed with the Herschel Space Observatory.

Methods. For each object, we modelled the spectral energy distribution from the ultraviolet to the sub-millimetre using the dedicated, aperture-matched DustPedia photometry and the Code Investigating GALaxy Evolution (CIGALE). The value of $f_{\text {abs }}$ was obtained from the total luminosity emitted by dust and from the bolometric luminosity, which are estimated by the fit.

Results. On average, $19 \%$ of the stellar radiation is absorbed by dust in DustPedia galaxies. The fraction rises to $25 \%$ if only late-type galaxies are considered. The dependence of $f_{\mathrm{abs}}$ on morphology, showing a peak for Sb-Sc galaxies, is weak; it reflects a stronger, yet broad, positive correlation with the bolometric luminosity, which is identified for late-type, disk-dominated, high-specific-starformation rate, gas-rich objects. We find no variation of $f_{\mathrm{abs}}$ with inclination, at odds with radiative transfer models of edge-on galaxies. These results call for a self-consistent modelling of the evolution of the dust mass and geometry along the build-up of the stellar content. We also provide template spectral energy distributions in bins of morphology and luminosity and study the variation of $f_{\text {abs }}$ with stellar mass and specific star-formation rate. We confirm that the local Universe is missing the high $f_{\text {abs }}$, luminous and actively star-forming objects necessary to explain the energy budget in observations of the extragalactic background light.
\end{abstract}

Key words. dust, extinction - infrared: galaxies - galaxies: photometry - galaxies: ISM - galaxies: evolution

\section{Introduction}

A common refrain in the literature states that dust, despite representing only a tiny fraction of the total baryonic content of galaxies, has a profound effect on their physics and appearance. Undoubtedly, dust has a major role in shaping their spectral energy distribution (SED), by absorbing starlight in the ultraviolet (UV), optical and near infra-red (NIR) ranges and re-emitting it

* DustPedia is a collaborative focused research project supported by the European Union under the Seventh Framework Programme (2007-2013) call (proposal no. 606824, PI J. I. Davies, http://www . dustpedia.com). The data used in this work is publicly available at http://dustpedia.astro.noa.gr at Mid and far infra-red (MIR and FIR, respectively) and submillimetre (submm) wavelengths (for a review, see Galliano et al. 2018). Observations from the Infrared Astronomical Satellite (IRAS; Neugebauer et al. 1984) provided the first means to attempt an estimate of the amount of radiation reprocessed by dust in local galaxies. Soifer \& Neugebauer (1991) used the total dust luminosity density from the 12 to $100 \mu \mathrm{m}$ luminosity functions of the IRAS bright galaxy sample and an estimate of the observed starlight luminosity density to assess that the infrared luminosity is about $30 \%$ of that from late-type stars. Thus, the ratio between the dust and bolometric - i.e. stars + dust - luminosities (a quantity we refer to as $f_{\text {abs }}$ in this work) would be $\approx 24 \%$. Xu \& Buat (1995) derived $f_{\text {abs }}$ in a more direct way, from a (very 
sparse) coverage of the UV-optical-FIR SED for each object in a sample of $135 \mathrm{UV}$-selected late-type galaxies (LTGs). The average value for their sample is $\left\langle f_{\text {abs }}\right\rangle=31 \pm 1 \%$. A similar approach was used by Popescu \& Tuffs (2002) for 28 LTGs in the Virgo cluster, with a better coverage of the UV-optical SED and FIR data extending to $170 \mu \mathrm{m}$, from the Infrared Space Observatory (ISO; Kessler et al. 1996). They obtained $\left\langle f_{\text {abs }}\right\rangle=24 \pm 2 \%{ }^{1}$. Thus, dust was found to be responsible for $1 / 3-1 / 4$ of the total bolometric luminosity of a galaxy, a result in agreement with estimates from the interstellar radiation field in our own Galaxy (Cox \& Mezger 1989).

The knowledge of $f_{\text {abs }}$ is relevant for radiative transfer (RT) studies. Encoded in the quantity is information on the mass of the grains, on their space distribution with respect to the photon sources, and, of course, on their absorption and emission properties. RT fits to the surface-brightness distribution of edge-on spiral galaxies (Xilouris et al. 1999; Bianchi 2007; De Geyter et al. 2014) have proved unable in most cases to predict the correct levels of FIR/submm emission (Bianchi et al. 2000; Baes et al. 2010b; de Looze et al. 2012). These, as we will see, for the typical dust-lane edge-ons correspond to $f_{\text {abs }} \approx 30-40 \%$. Instead, the emission predicted in the FIR is about a factor 2-4 smaller than observed (see Mosenkov et al. 2016, 2018, for the latest RT modelling). Different solutions to this energy balance problem have been proposed, involving more complex geometries than those inferred from fits to optical images (Popescu et al. 2000; Bianchi 2008; Saftly et al. 2015) or an enhanced dust emissivity in the FIR/submm with respect to the standard Milky Way (MW) properties (Alton et al. 2004; Dasyra et al. 2005). Yet, some edge-ons do not show the same problem (De Geyter et al. 2015; Mosenkov et al. 2018) and one might wonder if the issue is related to a dependence of $f_{\text {abs }}$ on other galactic properties.

A characterisation of $f_{\mathrm{abs}}$ in the local Universe also impinges upon studies of galaxy evolution. Provided that the galaxy sample is representative enough of the local Universe population, the luminosity-weighted $\left\langle f_{\text {abs }}\right\rangle$ should be analogous to the $f_{\text {abs }}$ that describes the wavelength-dependent specific luminosity density, or cosmic SED (CSED), at redshift zero. The integration of CSEDs from all epochs up to the current, results in the extragalactic background light (EBL). Since the first detection of the EBL in the FIR/submm from the satellite COBE (Puget et al. 1996; Hauser et al. 1998), it has become clear that a strong evolution of the FIR/submm luminosity (i.e. an increase of $f_{\mathrm{abs}}$ for CSEDs at $z>0$ ) is required (Franceschini et al. 1998, 2001). In contrast with the SEDs of local galaxies, the EBL implies that about half of the UV-optical photons have been absorbed by dust over cosmic times (i.e. $f_{\text {abs }} \approx 50 \%$; for the latest EBL estimates, see Driver et al. 2016a; Franceschini \& Rodighiero 2017).

The earlier determinations of $f_{\text {abs }}$ relied heavily on correction factors to derive the full luminosity over a certain spectral range from a few datapoints. In particular, the full energy output under the peak of dust emission up to $1000 \mu \mathrm{m}$ was derived from the wavelengths accessible to IRAS or ISO by extrapolating fits to the available data or interpolating up to ground-based submm/mm observations available for a few objects. These corrections could increase the observed dust luminosities from $40 \%$ (Soifer \& Neugebauer 1991; Xu \& Buat 1995) to $100 \%$ or above (Popescu \& Tuffs 2002). The need for these corrections has

This value is obtained from the full sample of Popescu \& Tuffs (2002) as displayed in their Fig. 2. However, in the abstract and conclusion the authors prefer to quote $\left\langle f_{\mathrm{abs}}\right\rangle=30 \%$, the mean for spirals of Hubble type later than Scd. been obviated by the advent of the Herschel Space Observatory (Pilbratt et al. 2010), and in particular by the submm coverage provided by the Spectral and Photometric Imaging Receiver (SPIRE; Griffin et al. 2010).

Using Herschel and ancillary data from Key Insights on Nearby Galaxies: A Far-Infrared Survey with Herschel (KINGFISH; Kennicutt et al. 2011), Skibba et al. (2011) derived the ratio between dust and stellar emission by directly integrating well sampled SEDs. Their tabulated results convert into $\left\langle f_{\text {abs }}\right\rangle=$ $29 \pm 2 \%$ for LTGs (52 objects). Similar integrations have been carried out by Davies et al. (2012) for the FIR-selected bright galaxy sample of the Herschel Virgo Cluster Survey, though neglecting the contribution to the SED of UV and MIR radiation. For the 69 LTGs in Davies et al. (2012) it is $\left\langle f_{\text {abs }}\right\rangle=$ $31 \pm 2 \%$. These results have been confirmed by Viaene et al. (2016), so far the most complete study in terms of spectral coverage of the SED and number of objects from a complete sample. For 239 spiral galaxies from the Herschel Reference Survey (HRS; Boselli et al. 2010b), Viaene et al. (2016) find an average $\left\langle f_{\text {abs }}\right\rangle=32 \pm 1 \%$, after fitting the observed SED with the Multiwavelength Analysis of Galaxy Physical Properties tool (MAGPHYS; da Cunha et al. 2008).

We have performed a similar analysis on the galaxies of the DustPedia project (Davies et al. 2017), including both LTGs and early-type galaxies (ETGs). DustPedia includes the vast majority of nearby $\left(v<3000 \mathrm{~km} \mathrm{~s}^{-1}\right)$, large (with an optical diameter of at least $1^{\prime}$ ) galaxies detected in their stellar component at $3.4 \mu \mathrm{m}$ and with available data from the Herschel Science Archive (Riedinger 2009). The main advantage of DustPedia over HRS and other Herschel-based samples lies in the dedicated, aperture matched, photometry that was carried out from UV up to submm wavelengths (Clark et al. 2018). Furthermore, DustPedia has more than twice the number of objects available in the HRS, allowing us to explore the dependence of $f_{\text {abs }}$ on a larger dynamic range of other observables and physical quantities. Also, the photometric coverage is slightly better: for the work described in the remainder of the paper, a mean of 21 photometric datapoints are available for each object, while in Viaene et al. (2016) the maximum number was twenty. Finally, DustPedia builds on the final characterisation of the observations from the Herschel Space Observatory, whose postoperation support ended in 2017; thus it constitutes the ultimate database on the local Universe before the next generation FIR/submm space missions.

The paper is organised as follows: in Sect. 2 we briefly describe the selection of the sample, the method used in determining $f_{\text {abs }}$ and the other data used in the analysis. The variation of $f_{\text {abs }}$ with morphological type is presented in Sect. 3, while Sect. 4 is devoted to a comparison with previous results. In Sect. 5 we illustrate the dependence of $f_{\text {abs }}$ on the bolometric luminosity, in particular for LTGs. In Sect. 6 we check that the results are not biased by the inclusion of edge-ons or AGN in the sample. An additional bonus of the analysis, the definition of SED templates, is shown in Sect. 7. The $f_{\mathrm{abs}}$, SEDs and other properties of DustPedia galaxies are compared to those at higher redshift in Sect. 8. Finally, we summarise our findings in Sect. 9.

\section{Data and analysis}

The fraction of energy absorbed by dust in a galaxy can be defined as the ratio between the luminosity emitted by dust and the bolometric (attenuated starlight+dust, equivalent to 
un-attenuated starlight) luminosity:

$f_{\text {abs }}=\frac{L_{\text {dust }}}{L_{\text {stars }}+L_{\text {dust }}}=\frac{L_{\text {dust }}}{L_{\text {bolo }}}=\frac{\int L_{\lambda}^{\text {dust }} \mathrm{d} \lambda}{\int L_{\lambda} \mathrm{d} \lambda}$,

where the luminosity density $L_{\lambda}$ can be derived directly from the observed flux density $F_{\lambda}$ (the SED) under the common assumption $L_{\lambda}=4 \pi D^{2} F_{\lambda}(D$ is the galaxy distance, though of this is irrelevant for $f_{\text {abs }}$ ). The integration is carried over for wavelengths $\lambda>0.0912 \mu \mathrm{m}$, the ionising radiation at shorter $\lambda$ being absorbed preferentially by the atomic gas. We have modelled the SED of most DustPedia galaxies using the CIGALE (Code Investigating GALaxy Evolution; Noll et al. 2009) software package $^{2}$. We provide here a few details of the sample selection and modelling, while a full description is presented in a companion paper Nersesian et al., in prep.).

\subsection{Sample selection and photometry}

The DustPedia photometric database consists of aperturematched flux estimates from the UV to the submm for a sample of 875 galaxies. In addition, IRAS and Planck photometry is provided in a supplementary dataset. We used all the bands where most of the contribution is either from stars or dust, that is from the Galaxy Evolution Explorer (GALEX; Morrissey et al. 2007) FUV up to the Planck $850 \mu \mathrm{m}$ band. We excluded the photometric entries that have significant contamination from a nearby galactic or extragalactic source, and those with serious artefacts in the imagery or insufficient sky coverage for a proper estimate of the target/sky levels. From the supplementary IRAS/Planck database, we excluded the flux density that might miss a significant contribution from extended emission (for details on the database and flagging codes, see Clark et al. 2018). Finally, we pruned all objects which, after the flux selection, resulted in no estimate at all around the peak of dust emission for $60 \leq \lambda / \mu \mathrm{m} \leq 500$, and those with an insufficient coverage of the stellar SED, that is with no remaining fluxes for $0.35 \leq \lambda / \mu \mathrm{m} \leq 3.6$.

In total, 51 objects were excluded because of a global contamination flag, in most cases due to a nearby galaxy. The exclusion might in principle bias our results against objects undergoing starbursting episodes due to strong interactions. Indeed, these objects lie on the high luminosity tail of the distribution, with an average luminosity at $3.4 \mu \mathrm{m}$ almost an order of magnitude higher than for the rest of the sample. Using the average results for higher-luminosity galaxies, however, we tested that the exclusion has not likely altered significantly the main conclusions of the work. Another ten objects were excluded because of insufficient SED coverage, after the photometry flagging. Among them is, for example, M 104 Sombrero (NGC 4594) which has all its FIR/submm images flagged because of insufficient map coverage for a proper determination of the sky level. The final sample is thus reduced to a total of 814 objects. Still, we have more than 15 available photometric datapoints per object for $94 \%$ of these galaxies.

\subsection{SED fitting}

CIGALE models the SED of a galaxy by choosing a variety of modules for the stellar, gas and dust emission, and for dust attenuation; observations are compared with a grid of models defined

2 Version 0.12.1, available at http: //cigale. lam.fr by the various parameters describing each module; the code imposes the energy conservation between the amount of radiation absorbed and emitted by dust (Noll et al. 2009; Roehlly et al. 2014). For our work we have used: the delayed and truncated star-formation history module, which was found to describe well both field and cluster spirals (Ciesla et al. 2016); a modified version of the standard starburst-like dust attenuation (Calzetti et al. 2000; Boquien et al. 2016); the Bruzual \& Charlot (2003) single stellar population module of solar metallicity, coupled to the Salpeter (1955) initial mass function; the default set for the nebular emission module, based on CLOUDY templates (Ferland et al. 1998; Boquien et al. 2016), with ionisation parameter 0.01 and all the ionising radiation absorbed by gas. The version of CIGALE we used includes a new dust emission module implemented by Nersesian et al. (in prep.): formally, this is similar to the available d12014 module, that uses an updated version of the Draine \& Li (2007) formalism (see also Ciesla et al. 2014). However, the dust emission templates are computed from The Heterogeneous dust Evolution Model for Interstellar Solids (THEMIS; Jones et al. 2017), DustPedia reference model, whose optical properties are firmly based on laboratory measurements of amorphous silicates, hydrocarbons and carbonaceous materials. By varying a set of nine parameters, we built a grid of about $8 \times 10^{7}$ models. The complete grid specifications and an analysis of the performances of the new themis dust emission module are presented in Nersesian et al. (in prep.).

After minimising the $\chi^{2}$ over the grid, CIGALE produces a best fit for the SED, for the various parameters describing the modules, and the physical quantities related to them. One could integrate numerically the fitted SED to obtain $L_{\text {dust }}, L_{\text {bolo }}$, and $f_{\text {abs }}$. The fitted SED might be considered as a reasonable, physically-motivated, interpolant between the datapoints; for galaxies with a coarser SED coverage, it could be a substitute for the correction factors used in the earlier works. However, parameters derived by minimising $\chi^{2}$ over a coarse grid might be prone to degeneracies. Thus, we prefer the Bayesian-like estimates computed by CIGALE from the likelihood of the models, which also allows for an estimate of the uncertainties. We used the likelihood-weighted mean and standard deviation for $L_{\text {dust }}$ and $L_{\text {bolo }}$, and derived from these our estimates for $f_{\text {abs }}$ and its uncertainty (which is mostly due to the uncertainty on $L_{\text {dust }}$ ).

Example SED fits are shown in Figs. 1 and 2 for LTGs and ETGs, respectively. The figures show galaxies with different photometric coverage and with good (i.e. at the peak of the $\chi_{\mathrm{r}}^{2}$ distribution, with $\left.0.3<\chi_{\mathrm{r}}^{2}<0.6\right)$ and poor $\left(\chi_{\mathrm{r}}^{2}>4\right)$ fits. The $\chi_{\mathrm{r}}^{2}$ distribution is shown in Nersesian et al. (in prep.) We find that only $\lesssim 5 \%$ of the sample have $\chi_{\mathrm{r}}^{2}>3$ (and $\lesssim 3 \%$ with $\left.\chi_{\mathrm{r}}^{2}>4\right)$. For the majority of these objects, the large $\chi_{\mathrm{r}}^{2}$ values are the result of a few photometric points deviant from the general SED trend, and the CIGALE output can still be used to derive $f_{\text {abs }}$, in particular when the Bayesian-like estimates are used, as we have done here. In fact, the global trends we will show in the rest of the work change very little if the worse fits are excluded. Thus, we decided to retain the full sample. In Appendix A we show the difference between the Bayesian estimates we used and those from the best fit. We also compare the values of $f_{\text {abs }}$ derived from CIGALE and those from other methods, in particular from the MAGPHYS fitting code (da Cunha et al. 2008), used by Viaene et al. (2016). In fact, for the limited scope of the current work, the dependence of the results on the chosen methodology (and dust model) is small.

In this work we have also used the Bayesian estimates and uncertainties for the stellar mass $\left(M_{\text {stars }}\right)$ and star-formation rate (SFR) from the CIGALE output. For all the extensive 

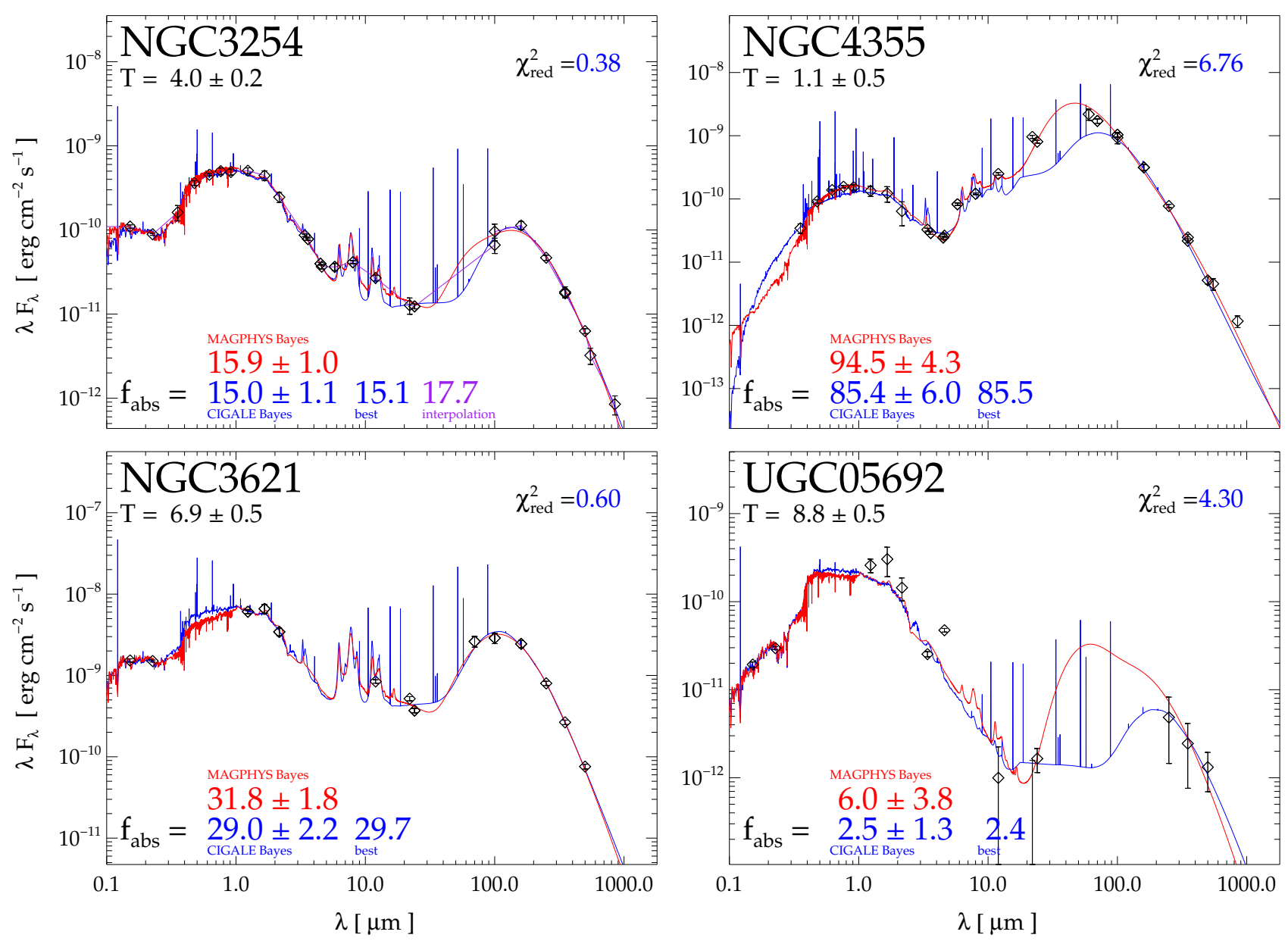

Fig. 1. Example SED fits for LTGs $(T>0.5)$. Top row: galaxies whose SEDs have a large band coverage ( $\geq 25$ datapoints), bottom row: those with a worse coverage ( $\leq 17$ datapoints). Galaxies on the left have fits with $\chi^{2}$ at the peak of its distribution $\left(0.3<\chi^{2}<0.6\right)$, those on the right a worse fit with $\chi^{2}>4$. The blue lines show CIGALE's best fit; both $f_{\text {abs }}$ Bayesian estimate (with uncertainty), used in the main text, and the estimate from best-fit parameters (i.e. the one describing the best fit itself) are reported. When available, we also show: in red, the MAGPHYS best fit (and Bayesian estimate of $f_{\text {abs }}$ ); in purple, the piece-wise SED used for galaxies with very good data coverage (and the estimate of $f_{\text {abs }}$ by direct integration of it). For the alternative estimates, see Appendix A.

quantities, we used the distances collected by Clark et al. (2018) and distributed together with the DustPedia photometry.

\subsection{Ancillary information}

As a morphology indicator, we take the Hubble stage $T$ obtained from the HyperLEDA database (Makarov et al. 2014) ${ }^{3}$. HyperLEDA $T$ 's might be non-integer, since, for most objects, they are the average over various estimates available in the literature. In the following, when we refer to a specific morphology type defined by an integer value of $T$, we will use all objects in the range $[T-0.5, T+0.5$; e.g. the Sa sample defined by $T=1$ include all objects with $0.5 \leq T<1.5$ ). Also, an estimate of the uncertainty is derived by HyperLEDA from the literature scatter.

Alternative morphology indicators are taken from Mosenkov et al. (2019). They fit a two-dimensional single Sérsic $(1963 ; 1968)$ surface brightness distribution to $3.4 \mu \mathrm{m}$ images of most DustPedia galaxies. They divide the sample into disk-like galaxies, characterised by Sérsic index $n<2$ ( $n=1$ is for exponential disks), and objects dominated by a

\footnotetext{
3 http://leda.univ-lyon $1 . \mathrm{fr} /$. Values were downloaded in mid-
} 2017 and are the same as those used by Mosenkov et al. (2019). spheroid (either spirals with dominant bulges or ellipticals) with larger values of $n$. While most late-type objects with $T>5$ are disk-like, objects classified in HyperLEDA as of earlier type show both disk-like and spheroid-like profiles. The Sérsic indices $n$ is available for almost the entire sample (810 out of 814 objects).

In order to test the effects of including edge-on galaxies in the analysis, we used the inclinations estimated by Mosenkov et al. (2019), which are more precise than those from HyperLEDA. They are available for most of the disk-like galaxies (446 out of 453 objects with $n<2$ ). We also used the mass of atomic gas, $M_{\mathrm{HI}}$, together with $M_{\mathrm{star}}$, as an indicator of the evolutionary stage of a galaxy. Through a literature search, $M_{\mathrm{HI}}$ has been retrieved for 709 objects (Casasola et al. and De Vis et al., in prep.).

\section{Dependence on morphological type}

In Fig. 3 we show $f_{\text {abs }}$ as a function of the Hubble stage $T$. We plot values (and error estimates on both axes) for each galaxy, using different colours for disk-dominated objects with Sérsic index $n<2$ (black circles) and spheroid-dominated with $n \geq 2$ (grey circles). We also plot results for bins of width $\Delta T=1$ centred on each (integer) $T$ value (see Sect. 2.3): the values 

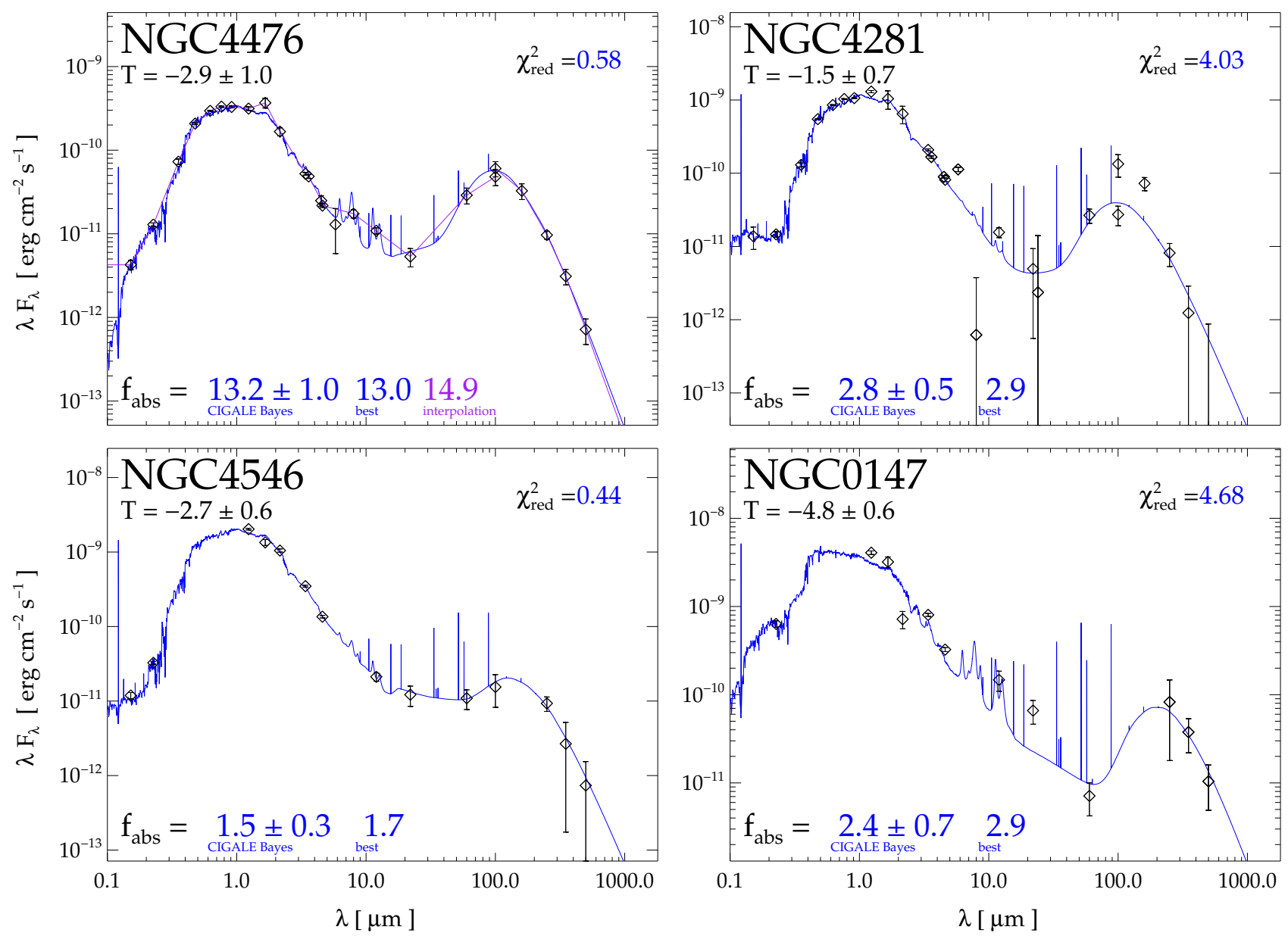

Fig. 2. Same as Fig. 1 but for ETGs $(T<0.5)$.

for the mean, standard deviation, median, lower $16 \%$ and upper $84 \%$ percentiles for each $T$ bin, as well as for broader bins in Hubble stage, are given in Table 1. For the full sample, we obtain $\left\langle f_{\text {abs }}\right\rangle=19.1 \pm 0.6 \%$. As expected, LTGs $(T \geq 0.5)$ are usually richer in dust content and have a higher average, $\left\langle f_{\text {abs }}\right\rangle=24.9 \pm 0.7 \%$, than ETGs $(T<0.5)$, for which $\left\langle f_{\text {abs }}\right\rangle=$ $7.4 \pm 0.8 \%$.

For LTGs, a mild trend with $T$ can be seen, though with a large scatter: $\left\langle f_{\text {abs }}\right\rangle$ is lower for $T=1-2$ objects $(\approx 25 \%)$, then it increases for $T=3-5$. The peak $\left\langle f_{\mathrm{abs}}\right\rangle$ is for $\mathrm{Sc}(T=5)$ galaxies, $34.0 \pm 1.6 \%$. For later types it decreases again, reaching a minimum for irregular galaxies $(T=10)$, with $\left\langle f_{\text {abs }}\right\rangle=12.3 \pm 1.6$. Despite the fact that the scatter in the $f_{\text {abs }}$ distribution is generally large, it appears to be smaller - relative to the absolute value - for types $4<T<7$. In this range there is a scarcity of (extreme) outliers and the mean and median are very similar. This moderate uniformity might be due to the nature of the objects, most of which are disk-dominated, as shown by Mosenkov et al. (2019). An average, disk-like, galaxy of this type is NGC 3621 (Fig. 1, bottom left). Later-type objects are still predominantly disk-like, yet the scatter in $f_{\text {abs }}$ increases, with a growing number of outliers on the lower side of the distribution. One such object is UGC 5692 (Fig. 1, bottom right).

The scatter is also larger for the earlier LTGs with $T=$ $1-3$, probably as a result of the increased number of spheroiddominated with respect to disk-dominated objects. Though spheroid-dominated galaxies can have both low and high $f_{\text {abs }}$ values, the eight DustPedia objects with $f_{\text {abs }}>70 \%$ are all bulge-dominated. The most extreme case is NGC 4355 with $f_{\text {abs }}=85 \pm 6 \%$ (Fig. 1, top right ${ }^{4}$ ). The IR emission of this galaxy (also known as NGC 4418) is powered by a compact starburst with a minor contribution from an AGN (Varenius et al. 2014). In Sect. 6 we show that the inclusion of AGN-hosts in our sample does not bias the results.

Going to ETGs, the relative scatter is higher for lenticular galaxies: $f_{\text {abs }}$ ranges from very low values, in most cases compatible with zero within the estimated uncertainty, up to the largest values observed for LTGs. The highest fractions of absorbed energy estimated in this morphology range are $f_{\text {abs }}=79 \pm 6$ and $72 \pm 11 \%$ for NGC $1482(T=-0.8)$ and NGC $1222(T=-2.6)$, two spheroid-dominated objects undergoing merger-driven starburst episodes (Vagshette et al. 2012; Young et al. 2018). An object with an average $f_{\text {abs }}$ is NGC 4476 shown in Fig. 2 (top left). As for Sa galaxies, in these morphology bins the $f_{\text {abs }}$ distribution is not Gaussian, the mean value being larger than the median. Also, there is no clear difference in $f_{\text {abs }}$ between the disk-dominated galaxies and the rest of spheroid-dominated objects for these morphologies. For elliptical galaxies, instead, the scatter in $f_{\text {abs }}$ is reduced and the number of objects with $f_{\text {abs }}$ larger than a few percent is smaller. We discuss the results for ETGs and the significance of the lowest $f_{\text {abs }}$ values later.

4 The fit for NGC 4355 is among the worst, because the upper end of the range we explored for the intensity of the interstellar radiation field is not high enough (for details, see Nersesian et al., in prep.). The $f_{\text {abs }}$ value we obtain is a lower limit to the true value. An alternative estimate (see Appendix A.3) yields $f_{\text {abs }} \approx 95 \%$, a value which is still marginally consistent with ours, within $1.5 \sigma$. 


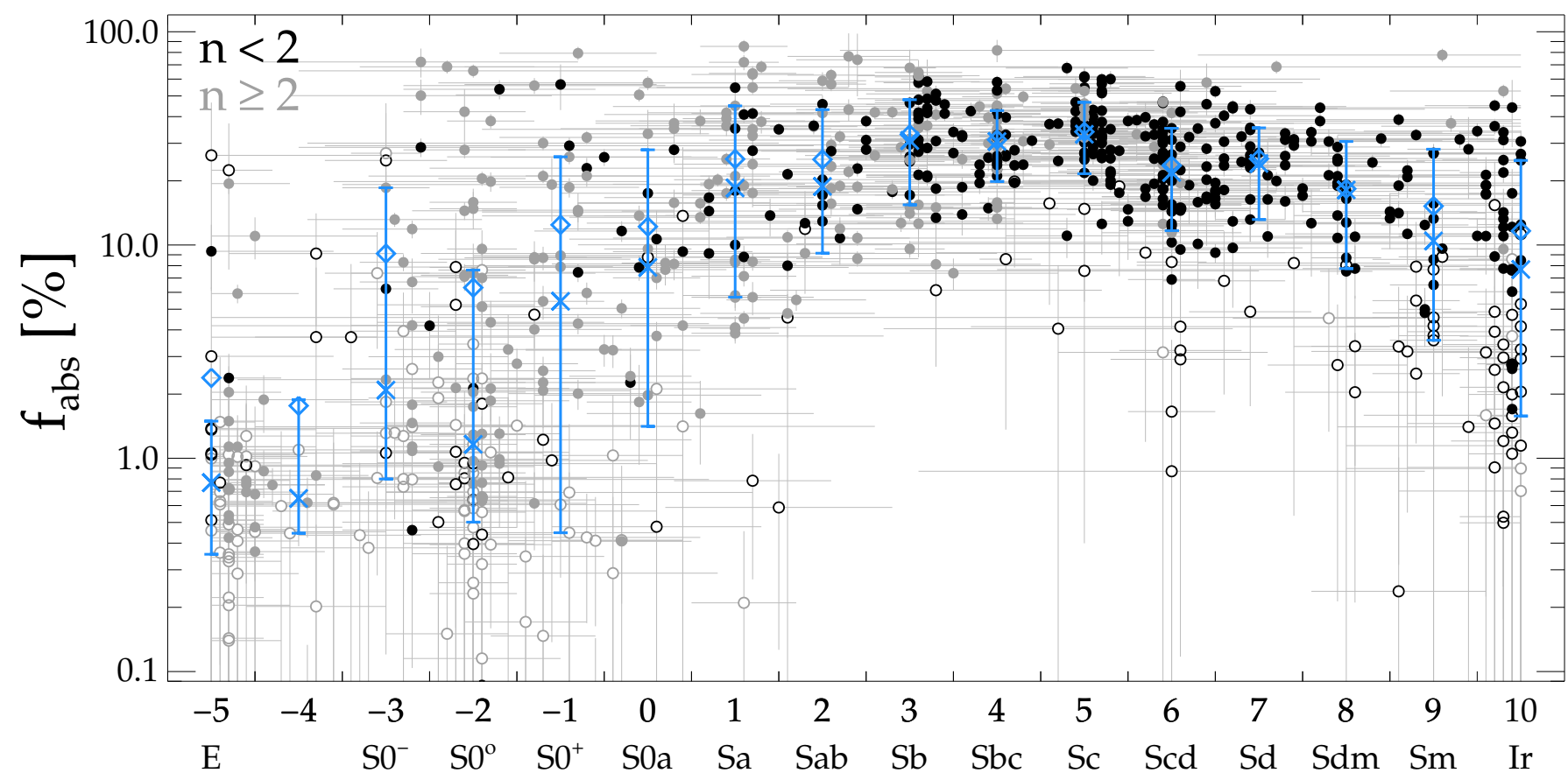

Fig. 3. $f_{\text {abs }}$ vs. Hubble stage $T$ for the DustPedia galaxies. For each bin in $T$, we plot the mean (diamonds), median (crosses) and the interval between the $16 \%$ and $84 \%$ percentiles (errorbars). Results on individual galaxies are also shown. Disk-dominated objects (those with fitted Sérsic index $n<2)$ are in black, spheroid-dominated $(n \geq 2)$ in grey. Open symbols are for galaxies with $f_{\text {abs }}$ estimate below $2 \sigma$. Errorbars are shown in light grey for all objects.

The subjective nature of the traditional morphology classification is reflected by the large uncertainty in the Hubble stage $T$, shown by horizontal error bars in Fig. 3. On average, the uncertainties are of the order of the bin width. There are, however, objects with uncertainties in $T$ spanning over several bins. This mixing might be responsible for part of the scatter in $f_{\text {abs }}$. Using the two-sample Kolmogorov-Smirnov (KS) test we found that in several cases there is a significant probability that the samples in nearby $T$ bins are drawn from the same distribution. For example, there is a probability of $74 \%$ that the $T=-1$ and 0 distributions are compatible with each other. In a few cases, the probability is large even for bins that are more separated: it is $93 \%$ for $T=0$ and 10 , and $56 \%$ for $T=2$ and 6 . Guided by Fig. 3 and by the results of the KS tests, we divided the sample into six larger morphology samples (see Table 1). The mean $f_{\text {abs }}$ value is the lowest for E galaxies; it increases from 9 to $25 \%$ going from $\mathrm{S} 0$ to $\mathrm{Sa}-\mathrm{Sab}$; it reaches a maximum for $\mathrm{Sb}-\mathrm{Sc}$ with $\left\langle f_{\text {abs }}\right\rangle \approx 33 \%$; it decreases again for later types, to $23 \%$ for Scd-Sm and $13 \%$ for Sm-Ir. The distribution of $f_{\text {abs }}$ in these larger samples are significantly different from each other, at a probability level $P<0.001$. The only exception are the Sa-Sab and Scd-Sdm bins, for which the KS test gives a probability of $10 \%$ of coming from the same distribution. This, and the KS results on some of the unit $T$ bins, indicates that $f_{\text {abs }}$ depends less on $T$ than on other physical properties of the samples.

\section{Comparison with previous estimates}

If we limit the comparison to Herschel-based works, we find that our result is marginally consistent, at a $2 \sigma$ level, with Skibba et al. (2011): for their whole late-type sample it is $\left\langle f_{\text {abs }}\right\rangle=29 \pm 2 \%$ vs. our $24.9 \pm 0.7 \%$. It is also consistent when only earlier spirals are included: it is $\left\langle f_{\text {abs }}\right\rangle=34 \pm 3 \%$ for their Sa-Sc $(0.5 \leq T<5.5)$ and $30 \pm 1 \%$ in our sample. As in Skibba et al. (2011), we find that in later type spirals dust absorbs a smaller fraction of stellar radiation. This is apparently at odds with the earlier ISO-based results of Popescu \& Tuffs (2002), where later types have higher $f_{\text {abs }}$. However, this might be simply due to the limited number of objects in their morphology bins and to the large corrections needed to take into account the submm spectrum not sampled by ISO. Yet, the average value for their full sample, $\left\langle f_{\text {abs }}\right\rangle=24 \pm 2 \%$, is consistent with our estimate. For ETGs, Skibba et al. (2011) have $\left\langle f_{\text {abs }}\right\rangle=25 \pm 8 \%$. Though their limited sample (ten objects vs. our 268) might be biased towards ISM-rich objects, the result is still marginally consistent at a $2 \sigma$ level, with our estimate, $\left\langle f_{\text {abs }}\right\rangle \approx 7.4 \pm 0.8 \%$.

A slightly higher estimate is obtained for LTGs by Davies et al. (2012), $\left\langle f_{\text {abs }}\right\rangle=31 \pm 2 \%$, at about $3 \sigma$ from our determination. This might be due to a series of causes: their sample is selected at $500 \mu \mathrm{m}$, biasing towards objects with higher dust emission fluxes; it is a cluster sample, including only Virgo galaxies (though there is no apparent change in the SED of cluster and field galaxies; Davies et al., in prep.); the integration procedure in their work is coarser and excludes a few wavelength ranges with significant stellar and dust emission. Nevertheless, the average value for the 7 ETGs where they measured $f_{\text {abs }}$ is $8 \pm 2 \%$, consistent with our estimate.

The $\left\langle f_{\text {abs }}\right\rangle$ estimate of Viaene et al. (2016) is also on the higher side. For their sample of 239 LTGs from HRS, they have $\left\langle f_{\text {abs }}\right\rangle=31.6 \pm 0.8 \%, 6 \sigma$ apart from the value found here. Since Viaene et al. (2016) is our reference work, for the completeness and dimension of their sample and the similarity of their method to ours, we investigated in details the reason for the discrepancy between the two analyses.

We first restricted the sample to the galaxies in common between Viaene et al. (2016) and this analysis. Because of the DustPedia selection rules, of the requirements imposed in this work (Sect. 2), and of the differences between the morphology classification used by HRS and DustPedia, they reduce to 197 objects. For them, we obtain $\left\langle f_{\text {abs }}\right\rangle=31.4 \pm 0.8 \%$ using the orig- 
Table 1. $f_{\text {abs }}$ values for DustPedia galaxies, for bins of $T$.

\begin{tabular}{cccccccc}
\hline \hline$T$ & Type & $N_{\text {obj }}$ & Mean & $\sigma$ & $16 \%$ perc. & Median & $84 \%$ perc. \\
\hline-5 & E & 51 & 2.4 & 5.4 & 0.4 & 0.8 & 1.5 \\
-4 & & 20 & 1.8 & 3.0 & 0.4 & 0.6 & 1.9 \\
-3 & $\mathrm{~S} 0^{-}$ & 34 & 9.1 & 15.6 & 0.8 & 2.1 & 18.6 \\
-2 & $\mathrm{~S}^{\circ}$ & 83 & 6.3 & 13.4 & 0.5 & 1.2 & 7.6 \\
-1 & $\mathrm{~S}^{+}$ & 43 & 12.4 & 17.3 & 0.4 & 5.4 & 25.9 \\
0 & $\mathrm{~S} 0 \mathrm{a}$ & 37 & 12.2 & 14.3 & 1.4 & 7.9 & 28.0 \\
1 & $\mathrm{Sa}$ & 50 & 25.3 & 21.1 & 5.7 & 18.5 & 44.9 \\
2 & $\mathrm{Sab}$ & 40 & 25.2 & 19.3 & 9.2 & 18.8 & 43.1 \\
3 & $\mathrm{Sb}$ & 58 & 33.0 & 15.5 & 15.4 & 30.9 & 48.0 \\
4 & $\mathrm{Sbc}$ & 63 & 31.2 & 12.9 & 19.9 & 30.6 & 42.7 \\
5 & $\mathrm{Sc}$ & 70 & 34.0 & 13.6 & 21.6 & 33.1 & 46.7 \\
6 & $\mathrm{Scd}$ & 84 & 23.6 & 12.5 & 11.7 & 21.9 & 35.3 \\
7 & $\mathrm{Sd}$ & 46 & 25.4 & 12.7 & 13.2 & 24.1 & 35.4 \\
8 & $\mathrm{Sdm}$ & 32 & 18.4 & 10.7 & 7.8 & 18.1 & 30.6 \\
9 & $\mathrm{Sm}$ & 36 & 15.2 & 15.2 & 3.6 & 10.4 & 28.1 \\
10 & $\mathrm{Ir}$ & 67 & 11.6 & 12.3 & 1.6 & 7.7 & 24.9 \\
\hline$[-5.0,-3.5]$ & $\mathrm{E}$ & 71 & 2.2 & 4.8 & 0.4 & 0.7 & 1.9 \\
{$[-3.5,0.5]$} & $\mathrm{S} 0$ & 197 & 9.2 & 15.0 & 0.6 & 2.3 & 18.6 \\
{$[0.5,2.5]$} & $\mathrm{Sa}-\mathrm{Sab}$ & 90 & 25.3 & 20.2 & 7.1 & 18.8 & 43.1 \\
{$[2.5,5.5]$} & $\mathrm{Sb}-\mathrm{Sc}$ & 191 & 32.8 & 13.9 & 19.2 & 31.1 & 46.1 \\
{$[5.5,8.5]$} & $\mathrm{Scd}-\mathrm{Sdm}$ & 162 & 23.1 & 12.4 & 10.8 & 21.2 & 34.8 \\
{$[8.5,10]$.} & $\mathrm{Sm}-\mathrm{Ir}$ & 103 & 12.9 & 13.4 & 2.1 & 8.6 & 26.7 \\
\hline$[-5.0,0.5]$ & ETGs & 268 & 7.4 & 13.5 & 0.5 & 1.4 & 14.3 \\
{$[0.5,10.0]$} & LTGs & 546 & 24.9 & 16.2 & 8.2 & 23.6 & 40.6 \\
{$[-5.0,10.0]$} & $\mathrm{All}$ & 814 & 19.1 & 17.4 & 1.1 & 15.6 & 36.2 \\
\hline & & & & & & & \\
\hline
\end{tabular}

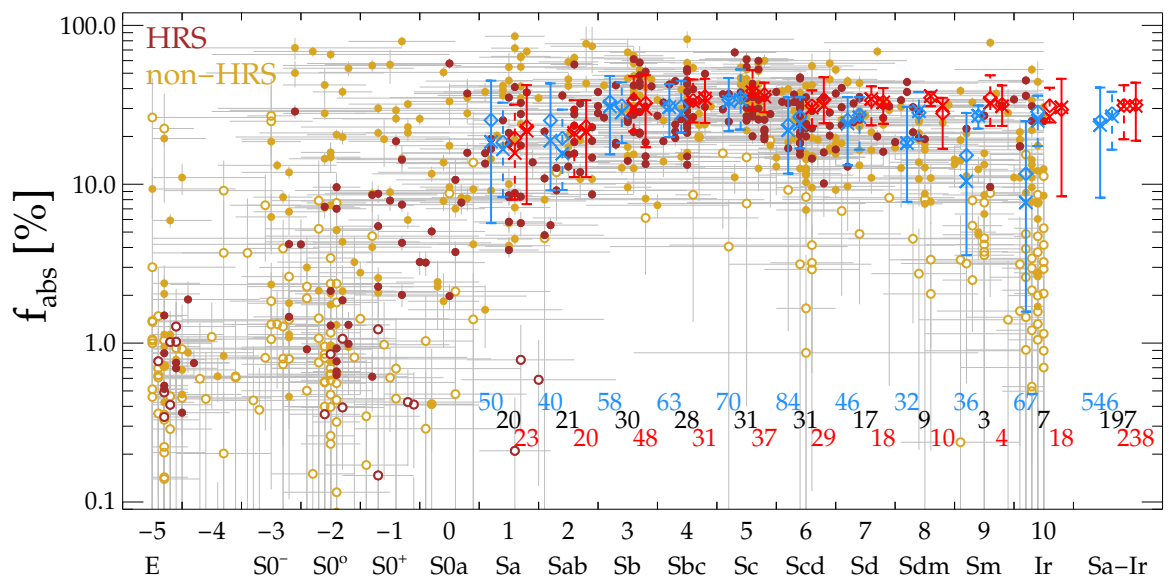

Fig. 4. As Fig. 3, but showing average results for: the DustPedia LTG sample (blue symbols with solid errorbars); the Viaene et al. (2016) analysis on HRS LTGs (red symbols with solid errorbars); the current analysis and the $f_{\text {abs }}$ values of Viaene et al. (2016) for a sample of galaxies in common between the two works (blue and red symbols with dashed errorbars, respectively). For the common sample, the DustPedia morphology is used. We also show results for each galaxy from the current analysis: brown symbols are for HRS objects included in DustPedia, yellow for additional DustPedia objects. The number of galaxies used in each analysis and for each bin is indicated. The Im and Pec classes of Viaene et al. (2016) are combined into the Ir, $T=10$, bin.

inal estimates from Viaene et al. (2016), very close to the value derived on their full sample. Instead, it is $\left\langle f_{\text {abs }}\right\rangle=27.9 \pm 0.8 \%$ using the results of our fits. The difference is thus reduced to $3 \sigma$, showing that part of the disagreement with Viaene et al. (2016) is due to the larger number and diversity of objects in DustPedia.

In Fig. 4 we plot the results of the averages described above as a function of $T$. For either the full HRS sample of Viaene et al. (2016), or the common HRS-DustPedia sample, there is little dependence of $\left\langle f_{\mathrm{abs}}\right\rangle$ on $T$ for the later type spirals with $T \gtrsim 6$. Instead, DustPedia includes many more low $f_{\text {abs }}$ objects in this morphology range, as can be seen by comparing the
DustPedia-only data points with those of the DustPedia-HRS (yellow vs. brown circles, respectively). The result is the decline of $\left\langle f_{\text {abs }}\right\rangle$ with $T$ we already described, together with the increased scatter in the distribution.

A detailed comparison between the photometry and models of Viaene et al. (2016) and those of the current work on the common DustPedia-HRS sample have shown that various reasons might be responsible for the residual discrepancy. A first explanation might reside in the different SED fitting code used by Viaene et al. (2016), MAGPHYS (da Cunha et al. 2008). Compared to DustPedia, more galaxies of the Viaene et al. (2016) 


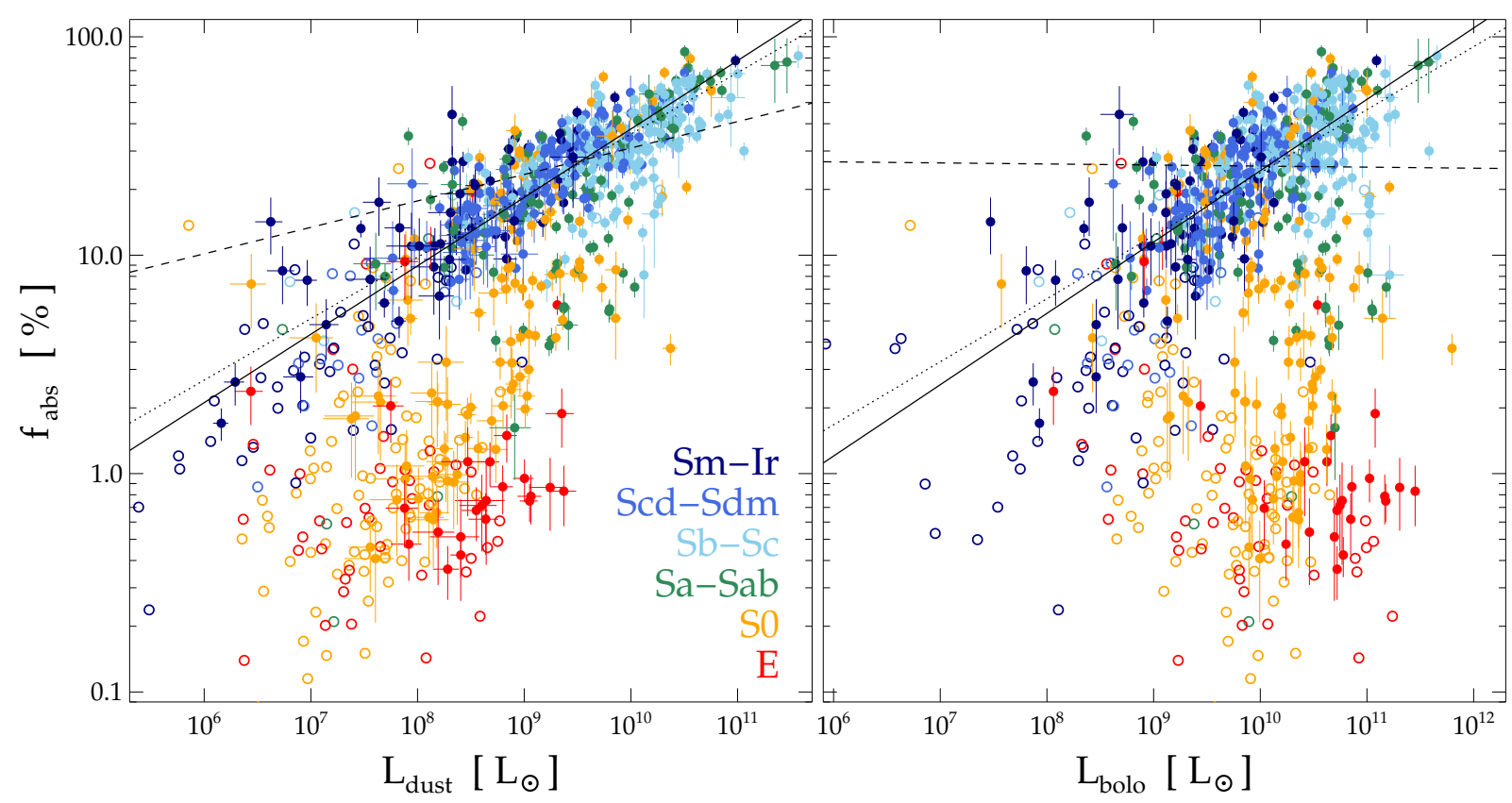

Fig. 5. $f_{\text {abs }}$ vs. $L_{\text {dust }}\left(\right.$ left panel) and vs. $L_{\text {bolo }}$ (right panel). Different colours refer to different morphological types. Open symbols without errorbars show $f_{\text {abs }}$ estimates below $2 \sigma$. Solid lines are the linear fits to datapoints for types later than Sb. Dashed lines refer to the intrinsic correlations produced by the uncertainties in the luminosity, under the assumption of no correlation of $f_{\text {abs }}$ vs. $L_{\mathrm{bolo}}$; dotted lines are the same, but assuming the same $f_{\text {abs }}$ vs. $L_{\text {bolo }}$ correlation as observed (see text for details).

sample do not have photometric constraints between 25 and $100 \mu \mathrm{m}$. This, coupled with the tendency of MAGPHYS to fill the gap with an unconstrained warm dust component (see Appendix A.3 and UGC 5692 in Fig. 1) tends to bias $f_{\text {abs }}$ to larger values. The bias is stronger for the few later type galaxies in common between the two samples, several of which showing an unconstrained SED on the Wien side of the thermal peak. Other, more systematic differences are due to photometry: the fluxes in Viaene et al. (2016) were not corrected for Galactic extinction (De Vis et al. 2017a); the FUV and NUV fluxes from HRS are smaller, and the Wide-field Infrared Survey Explorer (WISE; Wright et al. 2010) $12 \mu \mathrm{m}$ fluxes higher, than those from DustPedia (for details, see Clark et al.2018). All these differences concur, to various extent, in biasing $f_{\text {abs }}$ to (slightly) larger values.

In conclusion, our results are different from those of Viaene et al. (2016) in part because of the larger sample and diversity in DustPedia. When the same sample is used, there is a residual difference due to the methodology and photometry. The uniformity of the DustPedia photometry from the UV to the submm, combined with the more physical dust emission templates we have used in CIGALE, reassures us on the $f_{\text {abs }}$ estimates done in the current work.

\section{Dependence on luminosity}

In Fig. 5 we show $f_{\text {abs }}$ vs. $L_{\text {dust }}$ (left panel) and vs. $L_{\text {bolo }}$ (right panel). For LTGs, a positive trend (though with a large scatter) is clearly present: Sb-Sc objects appear to be clustered on the bright end of the trend and Sm-Ir objects on the faint one, though without a clear morphology division between intermediate luminosities. This indicates that luminosity is a more important factor than morphology in the determination of $f_{\text {abs }}$. The stronger dependence on luminosity rather than on morphology might also be the reason for the non-null result of the KS test between the Sa-Sab and Scd-Sdm distributions: while different in morphology, the two samples share a similar luminosity range. However, there are also significative departures from the trend: for types $\mathrm{Sb}-\mathrm{Sc}$, and more pronouncedly for $\mathrm{Sa}-\mathrm{Sb}$, a good fraction of bright galaxies have a lower $f_{\text {abs }}$. Going to ETGs, the majority of lenticular galaxies fall in the same low- $f_{\text {abs }}$, high-luminosity region, though several of them still share the main LTGs trend.The vast majority of ellipticals, instead, have low $f_{\text {abs }}$ and shows no correlation (with the exception of a few cases on the LTGs trend, possibly due to misclassified lenticulars).

\subsection{Trend for LTGs}

We quantify the strength of the correlation using the Kendall's correlation measure $\tau_{\mathrm{K}}$. For almost all the $\tau_{\mathrm{K}}$ measures presented in this work, the probability for the null hypothesis is small, at the $P<0.001$ level. For LTGs, we find $\tau_{\mathrm{K}}=0.47$ for $f_{\text {abs }}$ vs. $L_{\text {bolo }}$ and 0.63 for $f_{\text {abs }}$ vs. $L_{\text {dust }}$. The correlations improve to $\tau_{\mathrm{K}}=0.54$ and 0.66 , respectively, if only galaxies later than $\mathrm{Sb}(T \geq 2.5)$ are considered. In the following, we use this further morphological selection to define the trend, thus removing the low- $f_{\text {abs }}$, high-luminosity, Sa-Sab objects. We grouped the galaxies into four bins in $L_{\text {bolo }}$ (see Table 2 and Fig. 6). The mean steadily increases from $\left\langle f_{\text {abs }}\right\rangle=5.1 \pm 0.8 \%$ for $7.5 \leq \log _{10}\left(L_{\text {bolo }} / L_{\odot}\right)<8.5$ (where most of galaxies are disks of type Sm-Ir) to $38.6 \pm 1.5 \%$ for $\log _{10}\left(L_{\text {bolo }} / L_{\odot}\right) \geq 10.5$ (which are predominantly $\mathrm{Sb}-\mathrm{Sc}$ ). The scatter is reduced with respect to that for $T$ bins, confirming that $f_{\text {abs }}$ has a stronger dependence on $L_{\text {bolo }}$ than on morphology. Yet, the scatter is still large for the larger luminosities, since some high-luminosity $\mathrm{Sb}$-Sc galaxies stay on the low- $f_{\text {abs }}$, high-luminosity tail more common for the (removed) Sa-Sab objects. Linear fits (in log-log space) to the trends for $T \geq 2.5$ yield

$\log _{10}\left(f_{\text {abs }}[\%]\right)=-(1.9 \pm 0.2)+(0.33 \pm 0.02) \log _{10}\left(L_{\text {bolo }} / L_{\odot}\right)$,

and

$\log _{10}\left(f_{\text {abs }}[\%]\right)=-(1.6 \pm 0.1)+(0.31 \pm 0.01) \log _{10}\left(L_{\text {dust }} / L_{\odot}\right)$ 
Table 2. $f_{\mathrm{abs}}$ values for DustPedia galaxies later than $\mathrm{Sb}$, for bins of $L_{\mathrm{bolo}}$.

\begin{tabular}{cccccccc}
\hline \hline $\log _{10} L_{\text {bolo }} / L_{\odot}$ & $\left\langle L_{\text {bolo }} / L_{\odot}\right\rangle$ & $N_{\text {obj }}$ & Mean & $\sigma$ & $16 \%$ perc. & Median & $84 \%$ perc. \\
\hline$[7.5,8.5)$ & $1.6 \pm 0.9 \times 10^{8}$ & 30 & 5.1 & 4.6 & 1.2 & 3.1 & 8.6 \\
{$[8.5,9.5)$} & $1.7 \pm 0.8 \times 10^{9}$ & 104 & 13.1 & 8.7 & 3.8 & 11.9 & 21.3 \\
{$[9.5,10.5)$} & $1.2 \pm 0.8 \times 10^{10}$ & 216 & 27.4 & 11.1 & 16.4 & 27.1 & 37.3 \\
{$[10.5,11.5)$} & $6.2 \pm 3.4 \times 10^{10}$ & 97 & 38.6 & 14.6 & 25.0 & 38.3 & 54.3 \\
\hline
\end{tabular}

Notes. For $L_{\text {bolo }}$ we also give the mean and standard deviation within each bin.

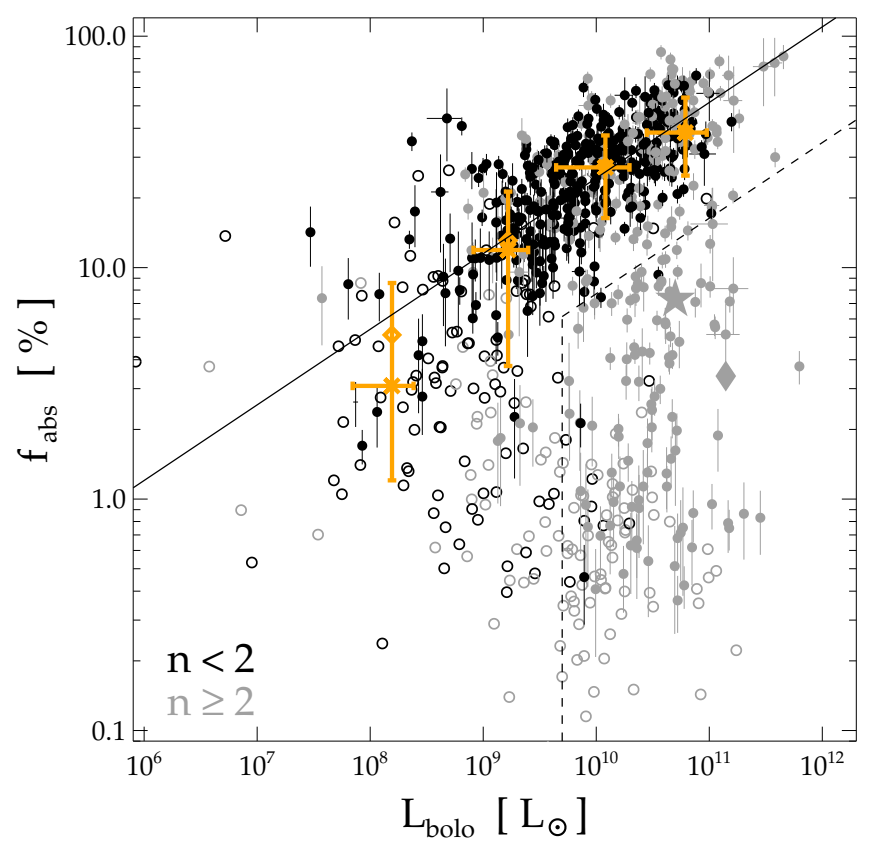

Fig. 6. $f_{\text {abs }}$ vs. $L_{\text {bolo }}$. Different colours are used for disk- and bulgedominated galaxies. Open symbols without errorbars show $f_{\text {abs }}$ estimates below $2 \sigma$. We plot in orange the mean (diamonds), median (crosses) and the interval between the $16 \%$ and $84 \%$ percentiles (errorbars), for each of the $L_{\text {bolo }}$ bins of Table 2 . The fit to the main trend is indicated, together with the area for the selection of the low- $f_{\text {abs }}$ tail. The star indicates M 31 and the diamond NGC 4594 (see text for details).

with errors estimated with a Monte Carlo bootstrap procedure, fitting a thousand random representations of the dataset (the fits are shown as solid lines in Fig. 5). Using the same procedure we found that datapoints where $f_{\text {abs }}$ is more than two times smaller than the estimated uncertainties (open symbols in Fig. 5, corresponding to $L_{\text {dust }} \lesssim 10^{8.5} L_{\odot}$ ), when considered together with their uncertainties, do not bias the Kendall's correlation measure (or the fit). The trend is also not significantly affected by the inclusion of the worse fits: $\tau_{\mathrm{K}}$ changes from 0.54 to 0.53 if only objects with $\chi_{r}^{2}<2$ are considered (432 out of the 456 galaxies of type later than $\mathrm{Sb}$ ).

In Fig. 6 we plot $f_{\text {abs }}$ vs. $L_{\text {bolo }}$ and distinguish among disk-dominated and spheroid-dominated galaxies, based on their Sérsic index. Apparently, most of the (high signal-to-noise ratio) $n<2$ objects align along the trend already discussed (with $\tau_{\mathrm{K}}=0.51$ ), while $n \geq 2$ are present both on the trend and on the lower $f_{\text {abs }}$ locus of ETGs. Thus, the use of the alternative morphology indicator has no impact in refining the sample and reducing the scatter in the observed trends. We also experimented with different cuts (and different morphology indicators, such as the bulge-to-total ratio, available for $\approx 64 \%$ of the sample; Mosenkov et al. 2019), but we were not able to find a better split between galaxies following the trend and galaxies outside of it.
If we consider only objects later than $\mathrm{Sb}$, and thus following the main trend, we find that disk dominated objects with $n<2$ have a lower $\left\langle f_{\text {abs }}\right\rangle$ than those with $n \geq 2$ : it is $23.5 \pm 0.7 \%$ vs. $32 \pm 2 \%$. However, this is the result of the bias in luminosity, since bulge-dominated objects have on average larger $L_{\text {bolo }}$ (in Fig. 6 this is shown by the larger number of grey symbols at the upper end of the luminosity range, and their lack at the lower end). When the analysis is conducted for smaller luminosity bins, such as those in Table 2, there is little difference between objects with $n$ below and above 2: for example, if we select all objects later than $\mathrm{Sb}$ with $10.5 \leq \log _{10}\left(L_{\text {bolo }} / L_{\odot}\right)<11.5$, it is $\left\langle f_{\text {abs }}\right\rangle=39 \pm 2 \%$ for $n<2$ and $38 \pm 3 \%$ for $n \geq 2$. The same is true for the third luminosity bin in Table 2 (there are few bulgedominated systems in the first bins for significative statistics).

\subsection{Significance of $f_{\mathrm{abs}}$ vs. $L_{\mathrm{bolo}}$}

Trends similar to that shown for $f_{\text {abs }}$ vs. $L_{\text {dust }}$ have been found in the past. The FIR luminosity was found to correlate with FIR to optical or UV ratios, using IRAS (Soifer et al. 1989; Wang \& Heckman 1996) and Spitzer data (Dale et al. 2009). Using Herschel observations of KINGFISH galaxies, Skibba et al. (2011) had shown a correlation consistent with our own. In particular, they noted the reduced fraction of absorbed radiation for later type dwarf and irregular galaxies with respect to earlier type spirals.

On the other hand, the correlation of $f_{\text {abs }}$ vs. $L_{\text {bolo }}$ has received less attention (though correlation with a related quantity, the stellar mass, had been noted; see Sect. 8.2). This correlation is potentially more interesting, as it denotes that dust absorption depends on the star-formation history of a galaxy. Indeed, we test in the following that the higher $\tau_{\mathrm{K}}$ for $f_{\text {abs }}$ vs. $L_{\text {dust }}$ does not imply that the correlation is more physically significant than that with $L_{\text {bolo }}$. The reason for this is that the luminosities shown in the $\mathrm{x}$-axis are used to derive $f_{\mathrm{abs}}$ on the $\mathrm{y}$-axis; spurious correlations are produced by the uncertainties in the luminosities and by the intrinsic scatter in $f_{\text {abs }}$.

Following De Vis et al. (2017a), we investigated the significance of the correlations using Monte Carlo procedures. In a first test, we assumed the null hypothesis, that is, that $f_{\text {abs }}$ is actually independent of the luminosity. For each object, we generated a mock value of $L_{\text {bolo }}$ assuming a Gaussian distribution centred on the measured value and with scatter provided by its uncertainty. We then extracted a random value of $f_{\text {abs }}$ (again assuming a Gaussian distribution, and the mean and scatter of the whole sample); from these, a new $L_{\text {dust }}$ was produced, to which we added a Gaussian scatter using as uncertainty a value randomly chosen from those associated with the 20 nearest, true, estimates of the dust luminosity. Finally, a mock value of $f_{\text {abs }}$ was derived from the ratio of the luminosities and linear fits (in log-log space) were derived. In the two panels of Fig. 5 we use dashed lines to show the average slopes obtained after iterating the procedure a thousand times. A faint, negative, correlation is found for $f_{\text {abs }}$ 
vs. $L_{\text {bolo }}$; negative, because $L_{\text {bolo }}$ appears in the denominator of the $f_{\text {abs }}$ estimate, and faint because the error estimates for $L_{\text {bolo }}$ are small. Instead, a significant spurious trend for $f_{\text {abs }}$ vs. $L_{\text {dust }}$ is present. In a second test, we verified that this bias is responsible for the larger $\tau_{\mathrm{K}}$ for $f_{\text {abs }}$ vs. $L_{\text {dust }}$. The procedure is almost identical to the previous one, with one exception: for each object, the random value of $f_{\text {abs }}$ is now derived by using the mean and scatter over the 20 objects which are nearest in the true estimates of the bolometric luminosity. We measured $\tau_{\mathrm{K}}$ for the two relations and repeated the procedure a thousand times. The average trends are shown with the dotted lines in Fig. 5 (both panels). The average correlations on the mock data are close to the fits to the true data (solid line), as much as the $\tau_{\mathrm{K}}$ values $(0.5$ and 0.65 for the mock $f_{\text {abs }}$ vs. $L_{\text {bolo }}$ and $f_{\text {abs }}$ vs. $L_{\text {dust }}$, respectively). For $f_{\text {abs }}$ vs. $L_{\text {bolo }}$ this is not unexpected, since in this second test we draw the mock $f_{\text {abs }}$ from the observed correlation. For $f_{\text {abs }}$ vs. $L_{\text {dust }}$, the test shows that the larger $\tau_{\mathrm{K}}$ is simply produced by the larger uncertainties in $L_{\text {dust }}$ and the scatter in $f_{\text {abs }}$.

\subsection{Outliers and ETGs}

Moving from LTGs to lenticulars, a larger fraction of bright bulgedominated galaxies move below the main trend. To investigate their nature, we selected galaxies with $L_{\text {bolo }} \geq 5 \times 10^{9} L_{\odot}$ and set an arbitrary upper limit of $f_{\text {abs }}$ at $3 \times$ below the fit to the main trend (dashed line in Fig. 6). While only $3 \%$ of Sb-Sc galaxies above the luminosity limit are included in the selection, the fraction grows to $30 \%$ for Sa-Sab galaxies and increases further to $78 \%$ for S0. Iconic objects that would fall in that locus of the plot are M 31, the Andromeda galaxy $\left(T=3, L_{\text {bolo }}=5 \times 10^{10} L_{\odot}\right.$ and $f_{\text {abs }}=7 \%$, estimated from the fit of Viaene et al. 2017) and M $104(T=1.1 \pm 3$, $L_{\text {bolo }}=1.4 \times 10^{11} L_{\odot}$ and $f_{\text {abs }}=3.4 \%$ from Skibba et al. 2011 ; similar estimates from the fits of de Looze et al. 2012). M 31 is not part of DustPedia, while M 104 was excluded from the sample (see Sect. 2.1): the two objects are shown with different markers in Fig. 6. As these two galaxies are characterised by a large bulge and a ring-like dust distribution, the different $f_{\text {abs }}$ behaviour of the other objects in the selection might in part be related to the different geometry of the stellar and dust distributions and to their evolution with respect to the objects along the trend. We discuss this issue further in Sect. 8.1.

A large proportion of ETGs have uncertain dust luminosities below $\approx 10^{8} L_{\odot}$. Indeed, $41 \%$ of S0 and $65 \%$ of ellipticals have $f_{\text {abs }}$ determinations below $2 \sigma$. Above that limit, some of the detections - and $f_{\text {abs }}$ determinations - might also be spurious. In particular for bright ellipticals, the target area is defined by optical images when instead the dust emission, when detected, comes from a much smaller area, often unrelated to the stellar distribution (Smith et al.2012b; di Serego Alighieri et al.2013). The aperturematched photometry in FIR/submm bands might in some cases be dominated by strong cirrus feature or background sources in the large target area, rather than true dust emission from the source. From a visual check of the FIR imaging of DustPedia ETGs, it is found that emission from the target itself is present in about a third of the ellipticals where $f_{\text {abs }}$ is estimated to be above $2 \sigma$ ( 8 out of 26 objects). An example of a spurious $f_{\text {abs }} \approx 2 \%$ determination is Andromeda's dwarf spheroidal NGC 147 (Fig. 2), where the uncertain Herschel-SPIRE detection in the FIR is due to the MW cirrus foreground (De Looze et al. 2016). Only a handful of ellipticals with true FIR emission detection have estimated $f_{\text {abs }} \lesssim 2 \%$ at a $>2 \sigma$ level. One of them is NGC 4487 (M 87), where the FIR emission is dominated by synchrotron radiation (Baes et al. 2010a) and not by dust. Since we only modelled the dust component, one might wonder if these low $f_{\text {abs }}$ estimates should be considered as simple upper limits. The contamination is present also for lenticulars, though to a lesser extent: about $78 \%$ of the $f_{\text {abs }} \geq 2 \sigma$ estimates are associated with true detections of FIR emission in the target (81 out of 104 galaxies). Nevertheless, the exclusion of the spurious $f_{\text {abs }}$ estimates does not produce a significant change in the statistics of Table 1, for both lenticulars and ellipticals.

Finally, we note that $f_{\text {abs }}$ in part depends on the colour of the radiation heating the dust. For the same stellar and dust distributions, ETGs and earlier-type spirals with typically redder stellar SEDs suffer less absorption than bluer LTGs, because of the dust attenuation law. This translates into different trends for $f_{\text {abs }}$ for galaxies where the younger stellar population dominates over the older one (Nersesian et al., in prep.).

\section{Checking the assumptions}

Our estimate of $f_{\text {abs }}$ from the observed SED relies on two main assumptions: the emission from both dust and stars is isotropic; stars are the only source of the radiation heating dust. We show in this section that neither the inclusion of edge-on galaxies nor AGN bias the results discussed so far.

\subsection{Dependence on inclination}

Isotropic emission is generally assumed in most estimates of luminosities. The assumption is certainly valid for objects showing a spherical symmetry for both dust and stars. For disk galaxies, it is generally assumed to be valid for the dust emission, since the ISM is optically thin in MIR to submm bands. However, it might be invalid in highly inclined objects, where a larger fraction of the stellar output could be absorbed along the disk plane.

RT models of edge-on galaxies (see Appendix B) suggest that the estimate of $f_{\text {abs }}$ decreases by $\approx 5-15 \%$ when passing from the edge-on to the face-on inclination; and $L_{\text {bolo }}$ increases by $\approx 30$ $40 \%$. Both effects are mainly due to the reduction of $L_{\mathrm{star}}$, as estimated from the output SED, in the edge-on case. Instead, we find little differences with inclination for DustPedia galaxies. When selecting disk-dominated objects, it is $\left\langle f_{\text {abs }}\right\rangle \approx 24 \pm 2 \%$ both for the edge-ons ( 75 objects with $i=90^{\circ}$ ) and the face-ons (69 objects with $i<40^{\circ}$, the limit chosen from the modelling of Appendix B to encompass little variations of the estimate with $i$ ). The KS tests shows that these two samples have $95 \%$ probability of coming from the same distribution. As a reference, the whole disk-dominated sample (446 galaxies) has $\left\langle f_{\text {abs }}\right\rangle=21.6 \pm 0.7 \%$. There might be a little bias towards face-on galaxies, which on average are $30 \%$ brighter than edge-on and the full sample - as indeed expected from the models; yet the estimates for the two limiting inclinations are the same within the errors.

The different behaviour with respect to inclination between the real estimates and the simulation results might in principle be due to a selection bias: the average galaxy was modelled from the mean results of De Geyter et al. (2014), which selected edge-ons with a prominent dust lane. The same is true for other edge-ons analysed via RT fitting. DustPedia includes ten edge-on galaxies with detailed fits ${ }^{5}$. They are all disk-dominated galaxies of type $\mathrm{Sb}-\mathrm{Sc}$, according to our criteria. For them, we obtain $\left\langle f_{\text {abs }}\right\rangle=36 \pm 4 \%$, higher than the average on the full disk

5 They are: NGC 891 (Xilouris et al. 1999), NGC 5907 (Xilouris et al. 1999; Mosenkov et al. 2018), NGC 5529 (Xilouris et al. 1999; Bianchi 2007; Mosenkov et al. 2018), NGC 4013 (Xilouris et al. 1999; Bianchi 2007; De Geyter et al. 2013; Mosenkov et al. 2018), IC 2531 (Xilouris et al. 1999; Mosenkov et al. 2016), NGC 4217 (Bianchi 2007; Mosenkov et al. 2018), NGC 4302, NGC 5746 (Bianchi 2007), NGC 4565 (de Looze et al. 2012), and IC 2461 (De Geyter et al. 2014). 
sample. However, these galaxies are brighter than average, with $10^{10} \lesssim L_{\text {bolo }} / L_{\odot} \lesssim 10^{11}$. If, among disk-dominated galaxies in the same luminosity range, we select the face-on cases $(40 \mathrm{ob}-$ jects with $i<40^{\circ}$ ) it is $\left\langle f_{\text {abs }}\right\rangle=32 \pm 2 \%$. Thus, there is no appreciable difference between the estimates of $f_{\text {abs }}$ on edgeon galaxies with well-defined dust lanes and face-on galaxies of analogous global properties. Results are identical if we select face-ons with $L_{\text {bolo }}$ larger by up to $50 \%$, to mimic the behaviour expected from RT models.

Since we found no evidence for a dependence of $f_{\text {abs }}$ on inclination, we considered edge-on galaxies together with the rest of the sample. In principle, a more isotropic behaviour of $f_{\mathrm{abs}}$ might be expected in galaxies where the energy budget is dominated by starlight from young clusters absorbed locally within parental clouds, an effect which is not captured by the smooth stellar and dust distributions adopted in the modelling of edge-on galaxies. A reduced $f_{\text {abs }}$ variation might be further concealed by the large observed scatter. However, edge-on DustPedia galaxies do show a difference with respect to face-ons: for a similar FIR SED, the stellar UV-to-NIR SED is redder (with a difference in NUV-r colour of $\approx 1.8$ magnitudes, as derived from the average SED of the galaxies analysed with RT fitting and that of faceons in the same luminosity range). Yet, for both inclinations, the energy exiting the galaxy as direct, attenuated, starlight, $L_{\text {stars }}$, is the same, relatively to $L_{\text {dust }}$ and the resulting $f_{\text {abs }}$ changes little. Investigating whether these effects are due to simplistic assumption in RT modelling of edge-ons, or to unforeseen selection rules in choosing their face-on counterparts, is beyond the scope of this paper. We hope to shed some light on these issues with dedicated RT modelling of the largest DustPedia galaxies (Verstocken et al., in prep. and Nersesian et al., in prep.) and of galaxies in large samples of DustPedia-like objects from cosmological simulations (Trčka et al., in prep.).

\subsection{AGN}

In a galaxy hosting a luminous AGN, the dust torus heated by the hard radiation from the accretion disk might dominate the MIR emission over that produced by the diffuse dust and stellar component. At least for the case of the bright AGNs seen at high- $z$, reprocessed MIR radiation from the torus could contribute significantly to the FIR-submm SED emission (Schneider et al. 2015; Duras et al. 2017). Even though such extreme objects are not likely to be present in a local sample, the host's dust might be directly exposed to the hard radiation from the nucleus, if its opening cone is not aligned with the disk axis. Thus, the AGN contribution might bias the estimate of $f_{\text {abs }}$ high with respect to the rest of the objects where stars are the dominant radiation source.

We have used the $90 \%$-confidence criterium of Assef et al. (2018), based on the WISE 3.4 and $4.6 \mu \mathrm{m}$ flux density, to ascertain which DustPedia galaxy might host a AGN. We find that 19 objects comply with that criterium. Among them are the record-holder NGC 4355 we already discussed; and NGC 1068, for which we are building detailed RT simulations to study the contribution of direct AGN radiation to dust heating (Viaene et al., in prep.). Several of these galaxies have high $f_{\text {abs }}$. However, they share the same trend as the other objects: for example, if we select the hosts of type later than $\mathrm{Sb}$ (6 objects), we find $\left\langle f_{\text {abs }}\right\rangle=43 \pm 16 \%$ for an average $L_{\text {bolo }} \approx 1 \times 10^{11} L_{\odot}$, a result compatible to that of the most luminous galaxies of the same type (See Table 2). This shows that the presence of an AGN does not alter significantly the energy balance in the SED.

For these reasons, we did not exclude AGN hosts from the analysis. A similar conclusion is reached by Viaene et al. (2016).

\section{Template SEDs}

Several templates of the stellar and dust SEDs have been produced in the past and used as tools to predict a galaxy's energy output and to study its evolution over cosmic time (see, e.g. Smith et al. 2012a; Ciesla et al. 2014; and references therein). As local benchmarks, here we provide templates based on our SED-fitting, which take advantage on the larger sample, broader spectral coverage and more homogeneous photometry of DustPedia galaxies with respect to previous work in the literature.

We derived spectral templates for the broad morphology bins of Table 1, and for the bins in $L_{\text {bolo }}$ defined in Table 2; in the second case, we use only the galaxies of type later than $\mathrm{Sb}$, which show a better defined trend of $f_{\text {abs }}$ vs. $L_{\text {bolo }}$, as we discuss in Sect. 5. For each galaxy sub-sample, templates were produced as follows: first, the SED of each galaxy was normalised to its $L_{\text {bolo }}$, to have all outputs on a similar scale; second, for each of the wavelength bins of CIGALE's best fit, the mean, median and percentiles of the specific flux densities were derived. The process was reiterated once to remove the $10 \%$ of the sample with the most deviant SEDs from the average ${ }^{6}$.

Figure 7 shows the average SEDs for each morphology bin. As expected from the $f_{\text {abs }}$ analysis, the scatter around each template is large. In particular for ETGs, the average template is significantly different from the median, being biased by a relatively large number of objects with strong FIR emission. As an indication of the scatter, the panels of Fig. 7 also include the individual SEDs of the $10 \%$ of each sub-sample which are most deviant from the average (which were however excluded from the template creation). Apparently, the scatter around the peak of optical-NIR radiation (at $\lambda \approx 1 \mu \mathrm{m}$ ) is smaller than that at the peak of FIR radiation (at $\lambda \approx 160 \mu \mathrm{m}$ ): the former ranges between 10 and $40 \%$, for ellipticals and Sm-Ir, respectively, the latter has a minimum at about $30 \%$ for $\mathrm{Sb}-\mathrm{Sc}$ galaxies and goes over $100 \%$ for ETGs. This might indicate that, in each morphology bin, there is a much larger variety in the dust emission spectrum than in the stellar; however, the reduced scatter in the optical results also from the normalisation of each spectrum on $L_{\text {bolo }}$, which is dominated in most cases (and in particular for ETGs), by the stellar output.

The average templates for each morphology bin are shown together in Fig. 8. A clear trend is seen in the UV/optical/NIR, with the SED becoming bluer from earlier to later types. This is a well known fact (see, e.g. Strateva et al. 2001). In the figure we compare our templates with the CSEDs for different morphological types derived by Kelvin et al. (2014) from 3727 galaxies $(0.025 \leq z \leq 0.06)$ in the volume-limited Galaxy And Mass Assembly (GAMA) survey. The CSEDs, obtained by integrating luminosity functions for the different Hubble types at different UV/optical/NIR bands, are very similar in shape to our determinations. This reassures us on the representativity of the DustPedia galaxies, despite the complexity of its sample definition.

In the FIR, our SEDs do not present a monotonous change with morphology, as foreseen from what is shown in Fig. 3: the relative contribution of dust to the bolometric luminosity strongly increases from ETGs up to $\mathrm{Sb}-\mathrm{Sc}$; then it decreases more gradually for the later types. The peak of dust emission for ellipticals is shifted to shorter wavelengths, implying warmer dust (though we should keep in mind the skewness of the SED distribution in Fig. 7). In analogy with Wien's displacement law and assuming modified blackbody emission and a typical MW

6 The templates are available at http://dustpedia.astro.noa.gr 

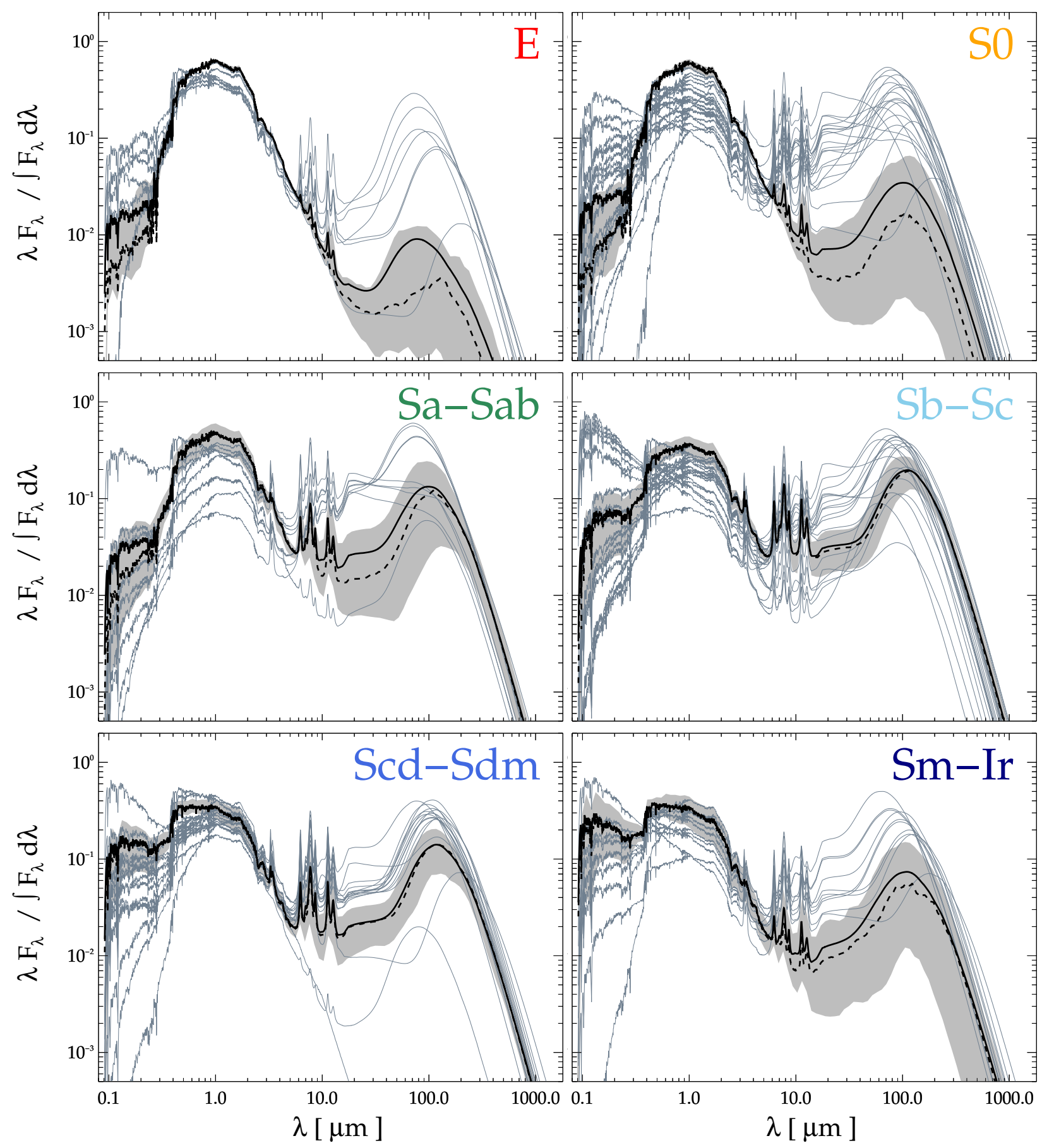

Fig. 7. Average (solid black lines) and median (dashed black lines) SEDs for the broad morphology bins defined in Table 1. For each sub-sample, we excluded from the template generation the $10 \%$ objects showing the most deviant SEDs (shown as grey lines). The shaded area delimits the 16 and $84 \%$ percentiles.

dust absorption cross section (see Sect. A.1), from the maximum of $\lambda \times F_{\lambda}$ we find $T_{\text {peak }}=34 \mathrm{~K}$ for ellipticals. Bluer FIR colours and hotter dust for ellipticals have been noted earlier (Boselli et al. 2010a; Davies et al. 2012; Auld et al. 2013) and attributed to the more intense radiation field in these galaxies, or to the additional contribution of electron collisional heating in the hot gas (Bocchio et al. 2013). For LTGs, the peak progressively (but moderately) moves to longer wavelengths from $\mathrm{Sa}-\mathrm{Sab}\left(T_{\text {peak }}=26 \mathrm{~K}\right.$, similar also to that for $\left.\mathrm{S} 0\right)$ to $\mathrm{Sb}-\mathrm{Sc}$ $\left(T_{\text {peak }}=24 \mathrm{~K}\right)$ and to $\operatorname{Scd}-\operatorname{Sdm}\left(T_{\text {peak }}=22 \mathrm{~K}\right)$. This might reflect a dependence of the dust temperature on $L_{\text {bolo }}$ (and thus on the intensity of the radiation field), whose average decreases going from $\mathrm{Sa}-\mathrm{Sab}$ and $\mathrm{Sb}-\mathrm{Sc}$ to Sc-Sdm. A dependence of the dust temperature on the infrared luminosity had been reported earlier for galaxies in large FIR surveys (Symeonidis et al. 2013; Magnelli et al. 2014). For the earlier type spirals (and the lenticulars) the hotter dust might result from the higher intensity of the heating radiation field in the presence of a strong bulge (Engelbracht et al. 2010). The later type bin (Sm-Ir), instead, does not follow the trend of LTGs and shows a bluer peak $\left(T_{\text {peak }}=24 \mathrm{~K}\right)$. In fact, dwarf galaxies tend to have warmer dust than more massive ones (see, e.g. Rémy-Ruyer et al. 2015).

We compared our templates with those derived by Ciesla et al. (2014) for the gas-rich HRS galaxies. They fitted 


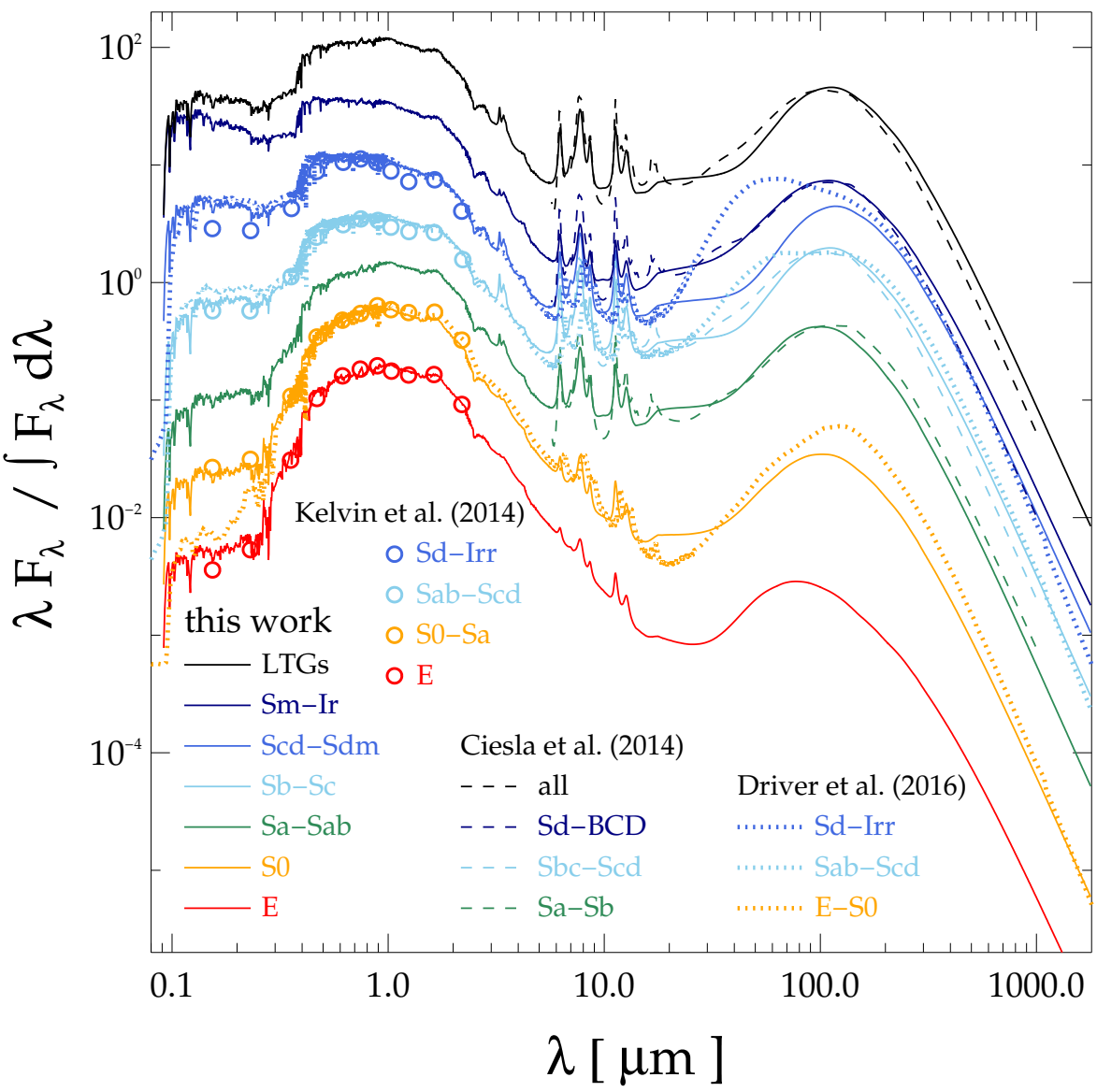

Fig. 8. Average templates of Fig. 7, shifted by an arbitrary factor for clarity. Also plotted are the templates of Ciesla et al. (2014; normalised to the same dust luminosity as ours) and the local CSEDs of Kelvin et al. (2014) and Driver et al. (2016b; normalised to $\lambda=$ $0.75 \mu \mathrm{m}$, i.e. the $i$-filter). We assigned the same colours to analogous morphology bins, even though the definition of the bins differs between references. the dust emission SED only, using CIGALE and the Draine et al. (2007) model. Their templates for various morphological bins are shown in Fig. 8, normalised to $L_{\text {dust }}$. We also compared their average template for the full sample with an average template derived for all DustPedia LTGs (black lines). Despite the differences in the sample, photometry, and dust models, the templates are rather consistent, with little variations. In particular, the template for their earlier-type bin peaks at longer wavelengths than ours, and thus does not show the trend with the later types we discussed; however, this is probably due to their inclusion of $\mathrm{Sb}$ into the bin, while our analysis of the mean $f_{\text {abs }}$ values made us conclude that objects of that morphology are similar to those of types Sbc and Sc. Our templates are also consistent, within the differences in modelling and morphology bin definition, with those obtained by Driver et al. (2016b) by median stacking FUVto-submm fits to the SED of GAMA galaxies with $z<0.06$. The major difference in this case is in the broader FIR SED and its peak at shorter wavelength; this is however due to the lack of MIR data and an unconstrained warm dust component introduced by the fitting tool they use, MAGPHYS (Sects. 4 and A.3).

We have seen in Sect. 5 that the stronger trend we found for $f_{\text {abs }}$ (and thus for the shape of the SED) is that with $L_{\text {bolo }}$ for galaxies later than $\mathrm{Sb}$. Fig. 9 shows the templates obtained after grouping these later type galaxies in the four bolometric luminosity bins defined in Table 2 . With respect to the templates for morphological bins, there is a slight improvement in the scatter, in particular for the dust emission spectrum, at least for the three brighter bins. The average templates are shown together in Fig. 10, after scaling them for the mean $L_{\text {bolo }}$ in each bin. The trend is clear, with templates becoming redder in the opticalUV and thus more extinguished and with more emission in the FIR as the luminosity increases. For the three higher luminosity bins, the peak of thermal emission shifts to shorter wavelengths with increasing $L_{\text {bolo }}$, with $T_{\text {peak }}$ increasing from $21 \mathrm{~K}$ to $24 \mathrm{~K}$. The average template of galaxies in the lower $L_{\text {bolo }}$ bin, instead, show a hotter temperature $\left(T_{\text {peak }}=29 \mathrm{~K}\right)$ and a broader FIR peak. Again with a caveat on the larger variety of objects in this bin (shown by the larger discrepancy between mean and median SED), the template is in agreement with the findings on dwarf galaxies, where the clumpier nature of the ISM and the reduced extinction result in hotter dust and a larger range of heating conditions (Rémy-Ruyer et al. 2015). The template SEDs binned in $L_{\text {bolo }}$ are compared with those from samples at higher redshift in the next section.

\section{DustPedia galaxies and the evolution of $f_{\mathrm{abs}}$}

The EBL spectrum is the summation of the SED of all galaxies, integrated over cosmic time. By considering separately the wavelength ranges dominated by stellar and dust emission, $f_{\text {abs }} \approx 50 \%$ can be estimated (using, e.g. the fitting models in Driver et al. 2016b; Franceschini \& Rodighiero 2017). The value is analogous to a luminosity-weighted average $f_{\text {abs }}$ for the whole galactic population in the Universe. The $\left\langle f_{\text {abs }}\right\rangle$ presented so far are un-weighted averages. It is $\left\langle f_{\text {abs }}\right\rangle \approx 20 \%$ for our full sample. It raises to $\approx 28 \%$ if we properly compute an $L_{\text {bolo-weighted value. The difference between the result on our }}$ local, DustPedia, sample and that from the EBL is yet another confirmation of the strong evolution in the fraction of stellar radiation reprocessed by dust (for a recent review including Herschel studies, see Lutz 2014). In this section, we compare our results for $z<0.01$ with those of surveys at higher redshifts to highlight the physical properties of the high- $f_{\text {abs }}$ objects which are underrepresented, or missing, in DustPedia. 

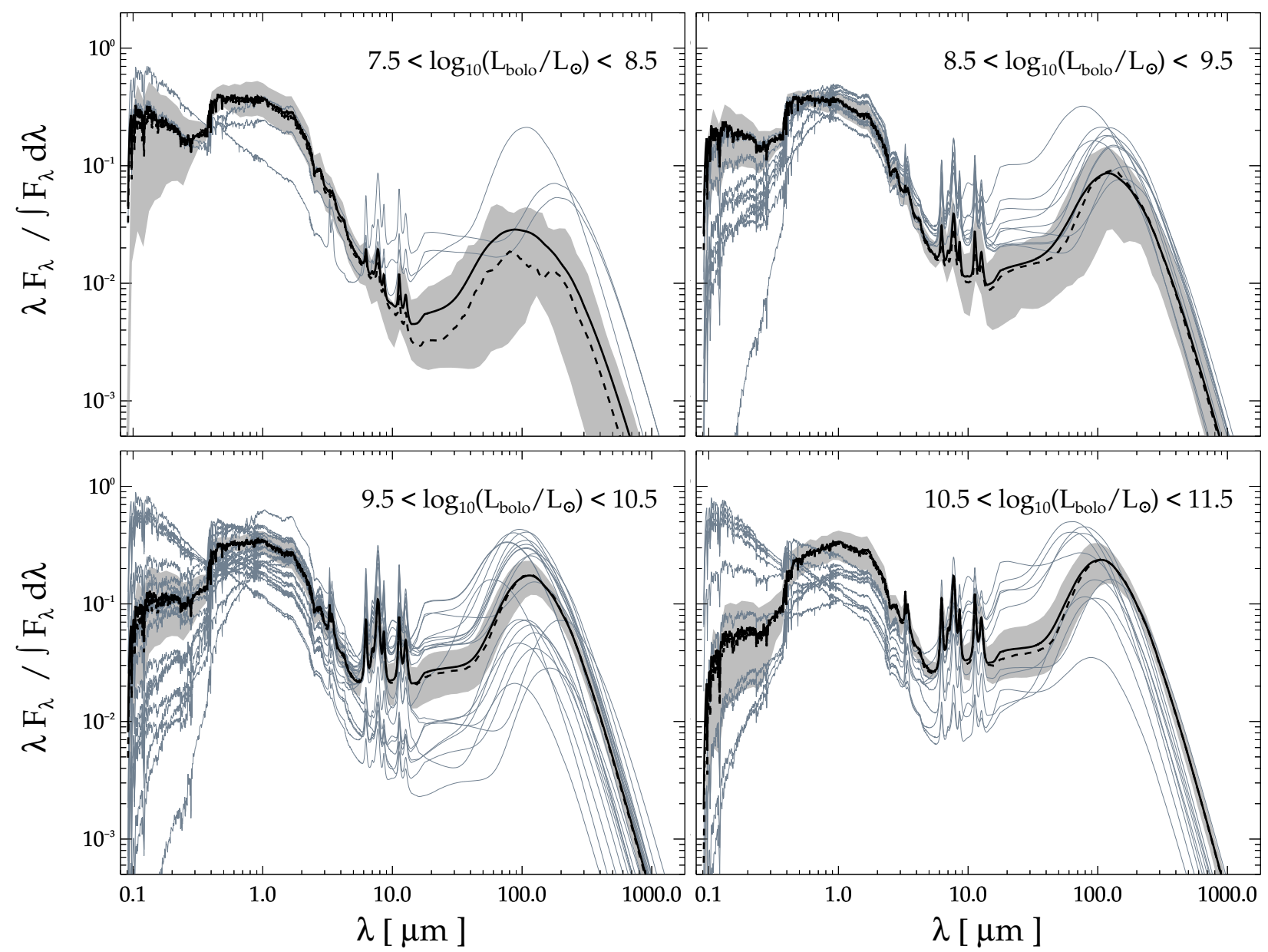

Fig. 9. Average (solid black lines) and median (dashed black lines) SEDs for four bins in $L_{\text {bolo }}$ defined in Table 2 for galaxies later than Sb. For each sub-sample, we excluded from the template generation the $10 \%$ objects showing the most deviant SEDs (shown by grey lines). The shaded area delimits the 16 and $84 \%$ percentiles.

\subsection{A simple evolutionary scenario}

It is shown that the evolution of the dust mass in a galaxy, relatively to the stellar (Cortese et al. 2012) or to the baryonic (gas+stars) mass (Clark et al. 2015; De Vis et al. 2017a), can be broadly described by relatively simple analytical models, taking into account the formation of dust seeds in asymptotic giant branch stars and supernovae, their destruction along with the astration process, and possibly with the inclusion of grain growth in the ISM and gas inflows and outflows (Edmunds 2001; Rowlands et al. 2014; De Vis et al. 2017b). In these studies, the gas fraction, $f_{\mathrm{gas}}=M_{\mathrm{HI}} /\left(M_{\mathrm{star}}+M_{\mathrm{HI}}\right)$ is used as a coarse indicator of the evolutionary stage of a galaxy. We investigate here if the evolution of the relative dust content with $f_{\text {gas }}$ is reflected by an analogous trend for $f_{\text {abs }}$, and if a simple evolutionary scenario can reconcile the difference between the local and EBL-derived $f_{\text {abs. }}$.

In Fig. 11 we show $f_{\text {abs }}$ vs. $f_{\text {gas }}$. Indeed a trend can be seen: objects where $f_{\text {gas }}$ is high, and thus in the earlier stages of their star-formation cycles, are preferentially of later type and lower $f_{\text {abs }} ; f_{\text {abs }}$ increases moving to earlier type spirals (Sb-Sc) with a smaller $f_{\text {gas }}$. A peak in the average $f_{\text {abs }}$ is reached at $f_{\text {gas }} \approx 0.1$. When considering only the galaxies up to this limit, the (negative) correlation has $\tau_{\mathrm{K}}=-0.34$. The evolution with morphological type is also reflected by that with the Sérsic index (not shown): most of disk-dominated systems are along the trend, the number of bulge-dominated ones increasing as the peak is reached. Earlier type, bulge-dominated objects have instead smaller $f_{\text {gas }}$ and their $f_{\text {abs }}$ dramatically reduces, though the abrupt change might be due to the neglect of molecular gas. Since gas-rich galaxies follow a well defined trend in Fig. 11, we used the selection $f_{\text {gas }}>0.1$ to try and improve the definition of the correlation of $f_{\text {abs }}$ vs. $L_{\text {bolo }}$ shown in Sect. 5 . We find $\tau_{\mathrm{K}}=0.53$, a value similar to those obtained by selecting galaxies later than $\mathrm{Sb}$, or disk-dominated galaxies.

A similar evolution is found when studying the variation of the dust mass with respect to the baryon mass (Clark et al. 2015; De Vis et al. 2017a). Objects with very high $f_{\text {gas }}$ are found to have a much smaller dust content than that extrapolated from scaling laws derived from more evolved galaxies; they have a high SFR per unit $M_{\text {star }}$ (specific star-formation rate, sSFR); models suggest that their radiation is absorbed near the starformation sites, where the initial seeds for dust grains also form (De Vis et al. 2017b; Rémy-Ruyer et al. 2015). As galaxies evolve and $f_{\text {gas }}$ decreases, the dust mass grows by accreting elements and/or molecules from the ISM onto the initial seed grains; the contribution of diffuse dust to the extinction increases (De Vis et al. 2017b). The dust mass, relative to the baryonic mass, reaches a peak at $f_{\text {gas }} \approx 0.5$, and then decreases when the dust destruction during the astration process dominates over the grain production and/or accretion (Clark et al. 2015; De Vis et al. 2017a).

The delayed peak of the $f_{\text {abs }}$ evolution at $f_{\text {gas }} \approx 0.1$ reflects the correlation of $f_{\text {abs }}$ with $L_{\text {bolo }}$ and must be due to the de- 


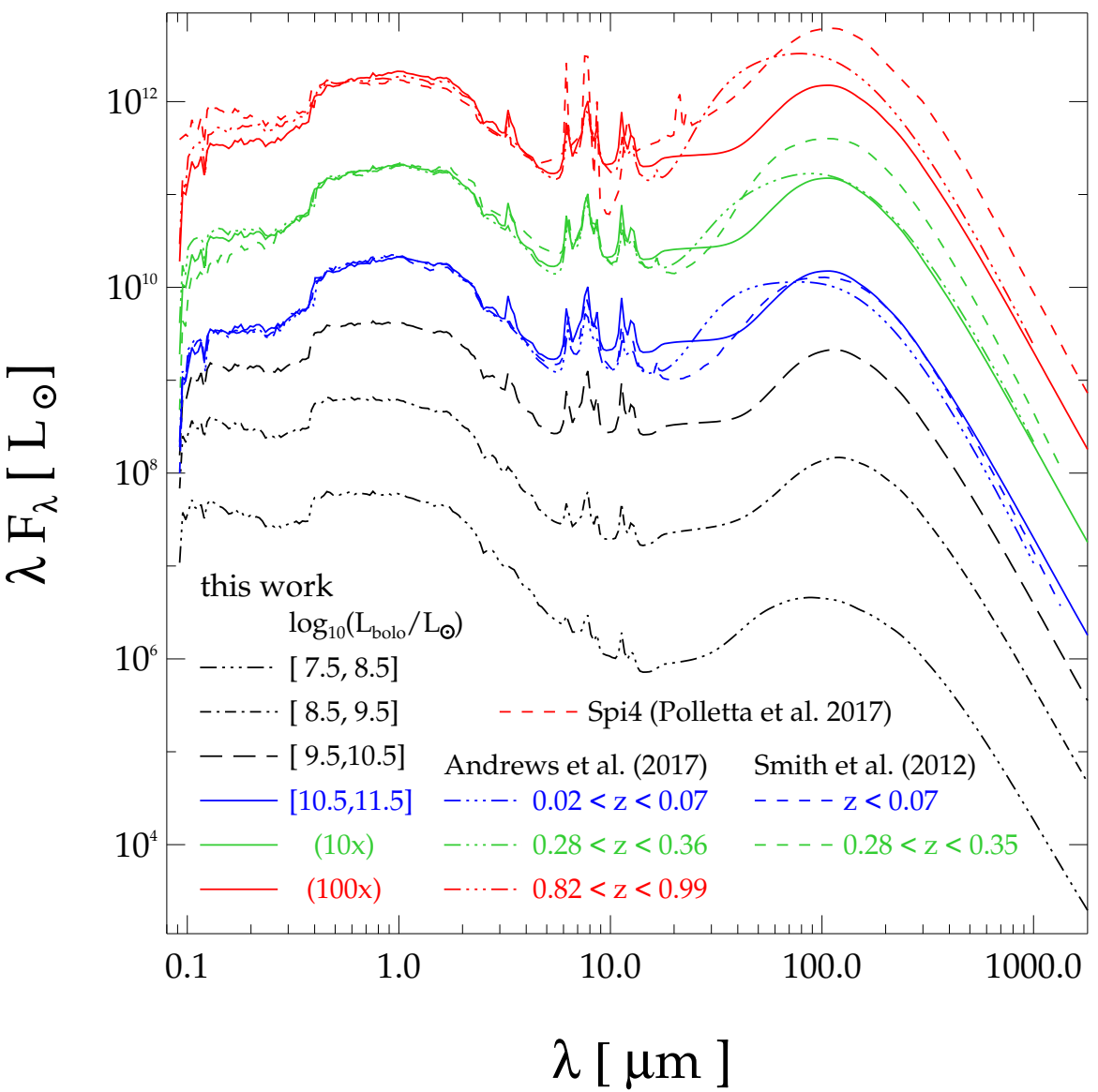

Fig. 10. Average templates of Fig. 9, scaled to the mean $L_{\text {bolo }}$ of each sub-sample. The highest$L_{\text {bolo }}$ template is shown three times, at the original scale (solid blue line) and multiplied by arbitrary factors (solid green and red lines). Also shown are two templates at different redshifts from Smith et al. (2012a), three CSEDs from Andrews et al. (2017), and the Spi4 template of Polletta et al. (2007). The SEDs from the literature have been normalised to the stellar luminosity of the highest- $L_{\text {bolo }}$ template.

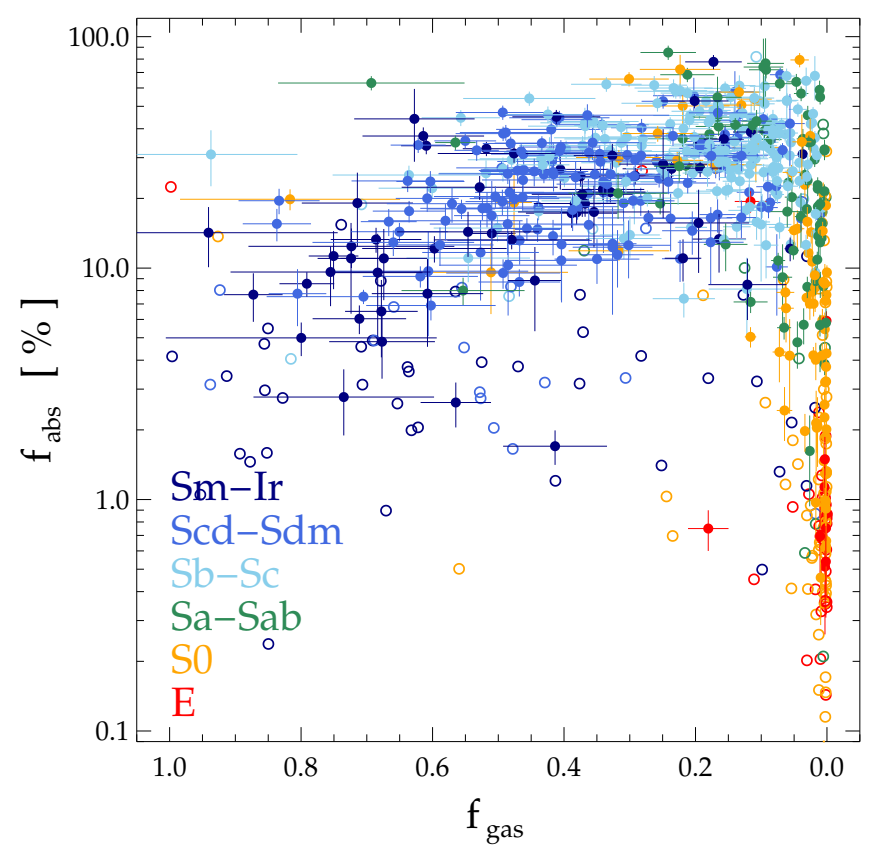

Fig. 11. $f_{\text {abs }}$ vs. $f_{\text {gas }}$. Same symbol and colour conventions as in Fig. 5 .

pendence of absorption on the absolute dust content (which is higher for higher $L_{\text {bolo }}$ ), and on its distribution with respect to the stars. Also, in high- $L_{\text {bolo }}$, high- $f_{\text {abs }}$ galaxies, the dust and/or star geometry might be more effective in absorption: an increasing contribution to absorption of the more pervasive diffuse medium is seen for objects with lower sSFR (De Vis et al. 2017a) which correspond, in our sample, to the more evolved objects.

Within this simple evolutionary picture, the precursor of current ETGs with $f_{\text {gas }} \leq 0.1$ must have passed through a phase which considerably reduced their dust mass. As a numerical experiment, we tried to simulate the conditions a step earlier in galaxy evolution by assigning to each of the objects with $f_{\text {gas }} \leq$ 0.1 a value of $f_{\text {abs }}$ derived from the galaxies with $f_{\text {gas }}>0.1$, assuming the trends of $f_{\text {abs }}$ with either $f_{\text {gas }}$ or $L_{\text {bolo }}$ (and their scatter). After the insertion of these mock values, the luminosityweighted $\left\langle f_{\text {abs }}\right\rangle$ of the sample rises from $\approx 28 \%$ to $40-45 \%$. Thus, while a simple monolithical scenario can in principle describe the evolution in the dust content of galaxies, it still falls short in predicting the evolution of $f_{\text {abs }}$ : a passive backward evolution from the DustPedia local sample does not explain the higher $f_{\mathrm{abs}}$ level implied by the EBL spectrum.

\section{2. $f_{\mathrm{abs}}, M_{\mathrm{star}}$ and sSFR for local and high-z objects}

Current models agree in assigning to star-forming disk galaxies the role of major contributors to the EBL; these objects undergo star-formation events driven by the infall of cold gas and dominate the star-formation history and infrared luminosity density for $z<$ 1; a minor contribution is expected from spheroids, whose peak in star-formation evolution - driven by merging events - occurs at higher redshifts (Franceschini et al. 2008, 2010; Domínguez et al. 2011; Driver et al. 2013; Franceschini \& Rodighiero 2017; Andrews et al. 2018).

Large-area multi-wavelength surveys including the FIR/submm, such as Herschel-ATLAS (Eales et al. 2010), can be used to study the dependence of $f_{\mathrm{abs}}$ on other physical 
properties and its evolution. Smith et al. (2012a) produced MAGPHYS fits to $H$-ATLAS extragalactic point sources detected at $250 \mu \mathrm{m}$ and derived template SEDs by median stacking. After the original publication, templates have been updated to include the successive releases of $H$-ATLAS data, totalling up to more than 12000 objects with redshift $z<0.35$ (Smith, priv. comm.; see also Rowlands et al. 2014). By binning SEDs according to $M_{\text {stars }}$ and sSFR, Smith et al. (2012a) find that the SED has a weak dependence on the former, while the latter governs the most striking changes, with an increasing contribution of dust emission for larger sSFRs. We used their templates to derive $f_{\text {abs }}$ and compare them to the values for the DustPedia sample.

The variation of $f_{\mathrm{abs}}$ as a function of $M_{\text {star }}$ and sSFR for DustPedia galaxies are shown in Fig. 12. Not surprisingly, the trend for $M_{\text {star }}$ shown in the left plot is similar to that for $L_{\text {bolo }}$ in Fig. 5, displaying a correlation for late-types $\left(\tau_{\mathrm{K}}=0.47\right.$ for objects later than Sb). A correlation of FIR/optical luminosity ratio with rotational velocity, and thus galactic mass, was already noted by Wang \& Heckman (1996) using IRAS data. Skibba et al. (2011) found a correlation with $M_{\text {star }}$ for the KINGFISH late and dwarf galaxies. Instead, Viaene et al. (2016) find none for HRS LTGs. The reason for this is in the smaller dynamic range in $M_{\text {stars }}$ for HRS (complete for $M_{\text {stars }} \gtrsim 8 \times 10^{8} M_{\odot}$; Eales et al. 2017). Indeed, if we select LTGs in common with HRS, we obtain $\tau_{\mathrm{K}}=0.06$ (the only estimate, among those presented here, with a non-null probability that the data are uncorrelated, $22 \%$ ). Instead, the KINGFISH sample, despite the smaller number of objects, has a sufficient variety as to include several lower luminosity later-type galaxies.

In the right panel of Fig. 12 we show $f_{\text {abs }}$ vs. the sSFR. There is a clear correlation between the two quantities for ETGs and spirals earlier than Scd, with $\tau_{\mathrm{K}}=0.66$. The correlation degrades to $\tau_{\mathrm{K}}=0.48$ when including the later type spirals and in particular the Sm-Ir galaxies, several of which show lower $f_{\text {abs }}$ for their high sSFR. The correlation was noted by both Skibba et al. (2011) and Viaene et al. (2016), though the latter missed the deviant behaviour of the dwarf galaxies, again because of the HRS flux limits. Motivated by Fig. 12, for objects with $\mathrm{sSFR} \geq 10^{-11} \mathrm{yr}^{-1}$ we derived $\tau_{\mathrm{K}}=0.53$ for $f_{\text {abs }}$ vs. $L_{\text {bolo }}$, not significantly different than the correlations obtained with the other selections discussed so far. Galaxies of type Sm-Ir align with the rest of LTGs (and with ETGs) if the SFR is normalised by the amount of gas available for star formation (i.e. a gas$\left.\mathrm{sSFR}=\mathrm{SFR} / M_{\mathrm{HI}}\right)$. They have a smaller gas-sSFR than the rest of LTGs (but higher than ETGs) and $\tau_{\mathrm{K}}=0.49$ for $f_{\mathrm{abs}}$ vs. gassSFR the full sample. We also experimented other correlations, such as $f_{\text {abs }}$ vs. $M_{\text {dust }}\left(\tau_{\mathrm{K}}=0.48\right.$ for types later than $\left.\mathrm{Sb}\right)$.

The templates of Smith et al. (2012a) do not show strong variations of $f_{\text {abs }}$ with $M_{\text {star }}$ until the largest masses, probably because of a selection bias (see later) and the reduced dynamic range in $M_{\text {stars }}$ (about two orders of magnitude). For $10.5 \leq$ $\log \left(M_{\text {stars }} / M_{\odot}\right)<11$, they have $f_{\text {abs }} \approx 50 \%$, considerably higher than what found in DustPedia for the same range, $33 \pm 3 \%$ (here and in the following, we select objects later than $\mathrm{Sb}$ ). This already implies an evolution in their sample, reaching $z \approx 0.35$. For the largest masses, $\log \left(M_{\text {stars }} / M_{\odot}\right) \geq 11$, their $f_{\text {abs }}$ reduces to $\approx 40 \%$. The reduction could be caused by the inclusion in the stacking of earlier type objects, which in DustPedia dominate over the range and have lower $f_{\text {abs }}$.

As anticipated, the templates of Smith et al. (2012a) binned in sSFR show strong changes in $f_{\text {abs }}$, from $\approx 30 \%$ for objects with $\log \left(\mathrm{sSFR} / \mathrm{yr}^{-1}\right)<-10.5$ to $\gtrsim 60 \%$ for $\log \left(\mathrm{sSFR} / \mathrm{yr}^{-1}\right) \geq-9.5$. In DustPedia, instead, we find $f_{\text {abs }}=28 \pm 3 \%$ for galaxies with
$-9.5 \leq \log \left(\mathrm{sSFR} / \mathrm{yr}^{-1}\right)<-9$. The value is biased low by the presence of the low- $f_{\text {abs }}$, high-sSFR, Sm-Ir objects. Values for $f_{\text {abs }} \gtrsim 60 \%$ can also be obtained for $\log \left(L_{\text {dust }} / L_{\odot}\right) \geq 11$ (from the templates of Smith et al. 2012a binned in this quantity). Actively star forming galaxies with these characteristics could align on the higher ends of the $f_{\text {abs }}$ vs. $L_{\text {dust }}$ (or $L_{\text {bolo }}$ ) trends of Fig. 5, and of the $f_{\text {abs }}$ vs. sSFR trends of Fig. 12 . With a presumably high $f_{\text {gas }}$, their position is instead expected to shift upward in the trend of Fig. 11. These galaxies are under-represented (or absent) in DustPedia but are necessary to match the energy budget of the EBL.

The evolution with redshift is clearly revealed by comparing the templates of Smith et al. (2012a) for local galaxies with $z<0.07$ and those for their highest redshift bin, $0.28<z<0.35$. They are shown in Fig. 10 together with our templates binned in $L_{\text {bolo }}$. If we exclude the unconstrained MIR range, the shape of their local template is remarkably close to that of our brightest bin, while that at higher redshift has a higher (and hotter) peak of thermal radiation; between these two redshifts, $f_{\text {abs }}$ increases from $\approx 40 \%$ to $60 \%$. The templates by Smith et al. (2012a) might be biased to higher $f_{\text {abs }}$ because of the selection at $250 \mu \mathrm{m}$. Indeed fainter levels of FIR emission, for similar redshift bins, characterise the CSEDs of Andrews et al. (2017); they were derived by summing-up MAGPHYS fits of about 300000 galaxies in the GAMA and COSMOS surveys, including objects detected and undetected in the FIR. Yet a progressive increase of $f_{\text {abs }}$ with $z$ can be seen, from $38 \%$ for $0.02<z<0.07$ (close to our brightest template, though again excluding the unconstrained MIR in their fits) to $45 \%$ for $0.28<z<0.36$, up to $60 \%$ for $0.82<z<0.99$ (see Fig. 10).

As we have discussed, the increase in $f_{\text {abs }}$ with $z$ might result from a larger fraction of luminous, star-forming galaxies - which are uncommon in DustPedia; this is consistent with the cosmic evolution of the SFR density and with the requirements set by the EBL. Apparently at odd with this, Domínguez et al. (2011) are able to predict the EBL with a sizeable contribution from normal star-forming galaxies. They use the pre-Herschel templates for local objects of Polletta et al. (2007) ${ }^{7}$, generated by the spectral evolution code GRASIL (Silva et al. 1998). The templates are used to fit UV to MIR SEDs in a sample of $0.2<z<1$ galaxies, and to predict their FIR; most of the objects are fitted by templates for spirals, among which the most successful is one named spi 4 (see Fig. 10). However, that template is not entirely representative of the galaxies sampled in DustPedia, since it has $f_{\text {abs }} \approx 70 \%$. Such high values are relatively rare in DustPedia, as there are just 14 galaxies with $f_{\text {abs }} \geq 60 \%$. Among them is the prototypical starburst M 82 (NGC 3034) for which we measured $f_{\text {abs }}=68 \pm 5 \%$. This is a further confirmation that the EBL is dominated by objects under-represented in our analysis.

Yet these $z<1$ objects do not represent the most extreme cases. Though not the dominant contributors to the local FIR background, submm galaxies (thought to be the precursors of local spheroid) have even higher values of sSFR and FIR luminosities, with almost all of their radiation processed by dust: from the median template of submm-galaxies with $z>1$ derived by Rowlands et al. (2014, see their Fig. 4), we estimated $f_{\mathrm{abs}} \approx 95 \%$.

\section{Summary and conclusions}

We used the code CIGALE to estimate the dust and bolometric luminosities, $L_{\text {dust }}$ and $L_{\text {bolo }}$, for 814 galaxies in the local

\footnotetext{
Available at http://www.iasf-milano.inaf.it/ polletta/ templates/swire_templates.html
} 


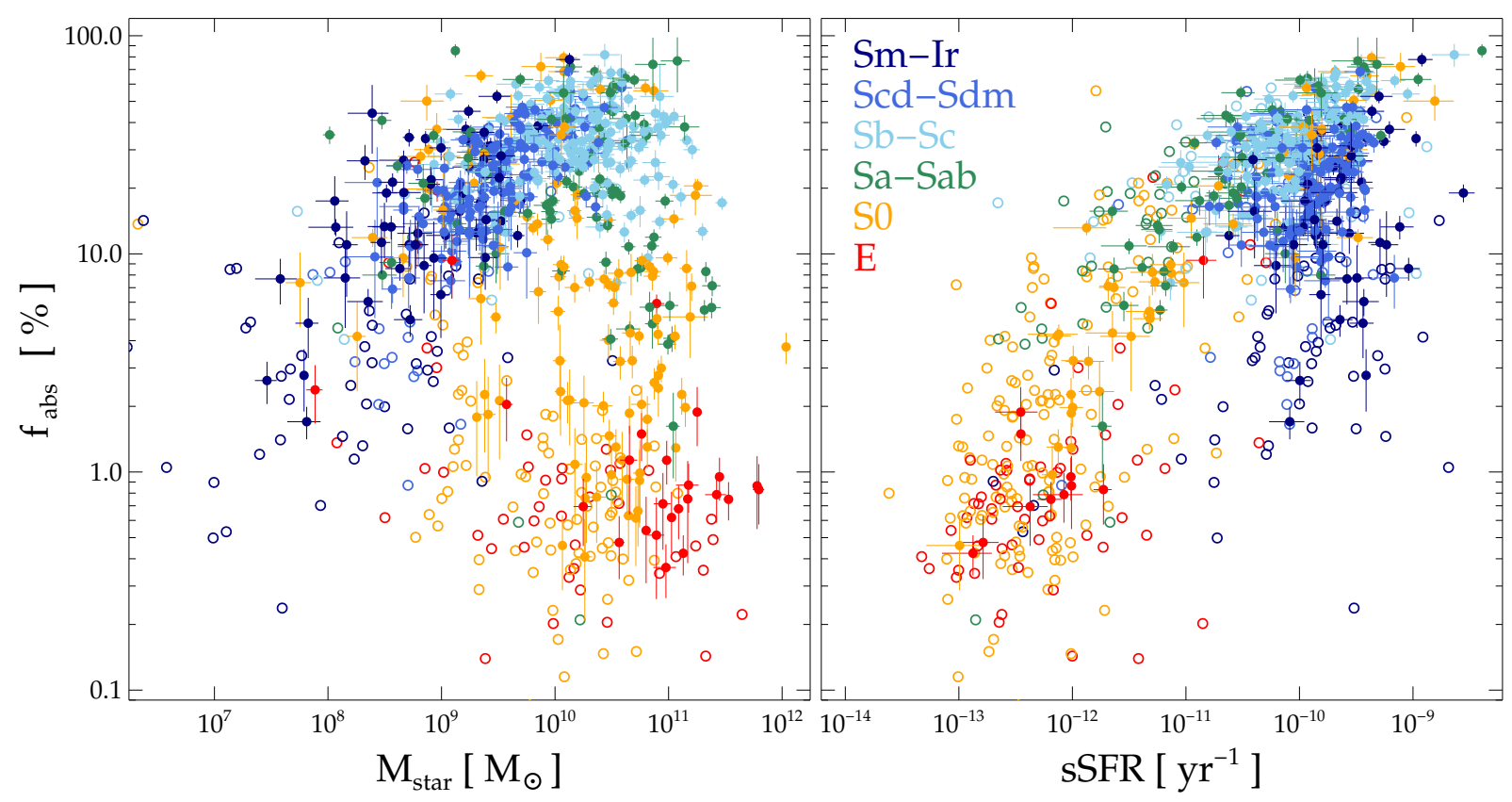

Fig. 12. $f_{\text {abs }}$ vs. $M_{\text {star }}$ (left panel) and specific SFR (right panel). Same symbol and colour conventions as in Fig. 5.

Universe. The objects of our study constitute the vast majority $(93 \%)$ of the DustPedia sample, for which an extensive coverage of the SED from the UV to the submm is available. From the luminosities we derive the fraction of radiation absorbed by dust, $f_{\text {abs }}$, and studied its dependence on a galaxy's morphological type, luminosity and other physical quantities. From the SED fits, we obtain averaged templates as a function of morphology and luminosity. Our main findings are:

- For the full sample, $\left\langle f_{\text {abs }}\right\rangle \approx 19 \%$, rising to $25 \%$ if only LTGs are considered. The LTG average is in line with previous determinations, though somewhat smaller. We argue that this is the result of the larger number and diversity of objects in DustPedia.

- A mild correlation is found between $f_{\text {abs }}$ and $L_{\text {bolo }}$, for galaxies of type later than $\mathrm{Sb}$, disk-dominated, gas-rich and with high specific star-formation rates. None of these specifications resulted in a significant improvement in the correlation: the scatter is large and the Kendall's correlation measure $\tau_{\mathrm{K}} \approx 0.5$ in all cases. A fit for objects later than $\mathrm{Sb}$ yields $f_{\text {abs }} \sim L_{\text {bolo }}^{1 / 3}$. Most of spheroid-dominated LTGs and of $\mathrm{Sa}-\mathrm{Sab}$ galaxies align on the same trend, as well as about a quarter of lenticulars.

- Similar correlations are seen between $f_{\text {abs }}$ and other physical quantities estimated by CIGALE, such as the mass of stars and the specific star-formation rate. Within our sample, a larger fraction of radiation is absorbed in LTGs where most of the build-up of stars and dust, and of the consumption of $\mathrm{H}_{\mathrm{I}}$ (or conversion into $\mathrm{H}_{2}$ ), has occurred.

- No apparent change in $f_{\text {abs }}$ with inclination is found, contrary to the predictions of RT models of edge-on galaxies.

- While $f_{\text {abs }}$ is higher for the higher luminosity LTGs in the sample $\left(\left\langle f_{\text {abs }}\right\rangle \approx 39 \%\right.$ for $\left.L_{\text {bolo }} \approx 10^{11} L_{\odot}\right)$, the fraction of absorbed radiation is not sufficient to explain the energy budget of the EBL, which requires $\left\langle f_{\text {abs }}\right\rangle \approx 50 \%$. DustPedia is missing the high luminosity and specific-star-formation-rate galaxies found in higher redshift samples, in agreement with the evolutionary picture that has emerged from observations and EBL modelling.
While it is not unexpected that intrinsically brighter galaxies have a larger dust content, it remains unclear why the fraction of absorbed radiation scales with the bolometric luminosity. Certainly, $f_{\text {abs }}$ must depend on several galactic properties: the star-formation history, the dust mass, the geometry of dust and stars, the relative proportion of radiation emitted in different environments, the optical properties of grains in each of them, to name a few. Indeed the scatter is large, yet correlations are clearly detected in our analysis. Explanation of these correlations will require a study of RT coupled with the evolution of a galaxy stellar, gas and dust content and structure.

Insights into these topics could be gained from RT models of large numbers of objects from cosmological simulations. Recently, Camps et al. (2016) have used the code Stellar Kinematics Including Radiative Transfer (SKIRT; Baes \& Camps 2015) to include the effects of dust in present-day mock galaxies from the Evolution and Assembly of GaLaxies and their Environments (EAGLE; Schaye et al. 2015) cosmological simulations; the general characteristics of local samples have been reproduced, both for what concern dust-extinguished optical radiation (Trayford et al. 2017) and dust infrared emission (Camps et al. 2016). Post-processed simulations for half a million EAGLE galaxies up to $z=6$ have been made available by Camps et al. (2018). An analysis of mock SEDs from that database along the lines of what has been done in the current work is yielding promising results: the trend $f_{\text {abs }}$ vs. $L_{\text {bolo }}$ seen in DustPedia galaxies is reproduced (Trčka et al., in prep.). We hope that the detailed knowledge of the input parameters will help in understanding the key physical properties drawing the correlation, and in studying the evolution of dust absorption and emission with cosmic time.

For a few open questions, the current resolution of largevolume hydrodynamical simulations might still be insufficient to describe the full range of ISM scales (such as the thickness of LTG disks; Trayford et al. 2017); in particular, a higher resolution might be needed to understand the apparent lack of anisotropy we found for $f_{\text {abs }}$, which could result from local dust extinction and emission at the scale of star-formation regions. 
In an exploratory study, Saftly et al. (2015) analysed a few hydrodynamical simulations of MW class objects and concluded that large-scale structure, such as spiral arms, might concur in hiding a considerable fraction of the dust mass from simplegeometry RT fits, an effect which is generally imputed to small-scale clumps (Popescu et al. 2000; Bianchi 2008). Modern higher resolution cosmological zoom-in simulations, such as those in the Numerical Investigations of Hundred Astrophysical Objects (NIHAO; Wang et al. 2015), or Auriga (Grand et al. 2017), to name a few, are already providing the ground to try to settle these issues and assess the combined effect of both small and large scales on a galaxy's dust emission and energy budget.

Acknowledgements. We thank M. Boquien and L. Ciesla for several discussions and help on the use of CIGALE, and an anonymous referee for helpful comments. IDL gratefully acknowledge the support of the Research Foundation Flanders (FWO Vlaanderen).

\section{References}

Alton, P. B., Xilouris, E. M., Misiriotis, A., Dasyra, K. M., \& Dumke, M. 2004, A\&A, 425, 109

Andrews, S. K., Driver, S. P., Davies, L. J. M., et al. 2017, MNRAS, 470, 1342

Andrews, S. K., Driver, S. P., Davies, L. J. M., Lagos, C. D. P., \& Robotham, A S. G. 2018, MNRAS, 474, 898

Assef, R. J., Stern, D., Noirot, G., et al. 2018, ApJS, 234, 23

Auld, R., Bianchi, S., Smith, M. W. L., et al. 2013, MNRAS, 428, 1880

Baes, M., \& Camps, P. 2015, Astron. Comput., 12, 33

Baes, M., Clemens, M., Xilouris, E. M., et al. 2010a, A\&A, 518, L53

Baes, M., Fritz, J., Gadotti, D. A., et al. 2010b, A\&A, 518, L39

Bianchi, S. 2007, A\&A, 471, 765

Bianchi, S. 2008, A\&A, 490, 461

Bianchi, S., Davies, J. I., \& Alton, P. B. 2000, A\&A, 359, 65

Bianchi, S., Giovanardi, C., Smith, M. W. L., et al. 2017, A\&A, 597, A130

Bocchio, M., Jones, A. P., Verstraete, L., et al. 2013, A\&A, 556, A6

Boquien, M., Kennicutt, R., Calzetti, D., et al. 2016, A\&A, 591, A6

Boselli, A., Ciesla, L., Buat, V., et al. 2010a, A\&A, 518, L61

Boselli, A., Eales, S., Cortese, L., et al. 2010b, PASP, 122, 261

Bruzual, G. 2007, ASP Conf. Ser., 374, 303

Bruzual, G., \& Charlot, S. 2003, MNRAS, 344, 1000

Calzetti, D., Armus, L., Bohlin, R. C., et al. 2000, ApJ, 533, 682

Camps, P., Trayford, J. W., Baes, M., et al. 2016, MNRAS, 462, 1057

Camps, P., Trčka, A., Trayford, J., et al. 2018, ApJS, 234, 20

Chabrier, G. 2003, PASP, 115, 763

Charlot, S., \& Fall, S. M. 2000, ApJ, 539, 718

Ciesla, L., Boquien, M., Boselli, A., et al. 2014, A\&A, 565, A128

Ciesla, L., Boselli, A., Elbaz, D., et al. 2016, A\&A, 585, A43

Clark, C. J. R., Dunne, L., Gomez, H. L., et al. 2015, MNRAS, 452, 397

Clark, C. J. R., Verstocken, S., Bianchi, S., et al. 2018, A\&A, 609, A37

Cortese, L., Ciesla, L., Boselli, A., et al. 2012, A\&A, 540, A52

Cox, P., \& Mezger, P. G. 1989, A\&AR, 1, 49

da Cunha, E., Charlot, S., \& Elbaz, D. 2008, MNRAS, 388, 1595

Dale, D. A., Cohen, S. A., Johnson, L. C., et al. 2009, ApJ, 703, 517

Dasyra, K. M., Xilouris, E. M., Misiriotis, A., \& Kylafis, N. D. 2005, A\&A, 437, 447

Davies, J. I., Bianchi, S., Cortese, L., et al. 2012, MNRAS, 419, 3505

Davies, J. I., Baes, M., Bianchi, S., et al. 2017, PASP, 129, 044102

De Geyter, G., Baes, M., Fritz, J., \& Camps, P. 2013, A\&A, 550, A74

De Geyter, G., Baes, M., Camps, P., et al. 2014, MNRAS, 441, 869

De Geyter, G., Baes, M., De Looze, I., et al. 2015, MNRAS, 451, 1728

de Looze, I., Baes, M., Bendo, G. J., et al. 2012, MNRAS, 427, 2797

De Looze, I., Baes, M., Bendo, G. J., et al. 2016, MNRAS, 459, 3900

De Vis, P., Dunne, L., Maddox, S., et al. 2017a, MNRAS, 464, 4680

De Vis, P., Gomez, H. L., Schofield, S. P., et al. 2017b, MNRAS, 471, 1743

di Serego Alighieri, S., Bianchi, S., Pappalardo, C., et al. 2013, A\&A, 552, A8

Domínguez, A., Primack, J. R., Rosario, D. J., et al. 2011, MNRAS, 410, 2556 Draine, B. T., \& Li, A. 2007, ApJ, 657, 810

Draine, B. T., Dale, D. A., Bendo, G., et al. 2007, ApJ, 663, 866

Driver, S. P., Robotham, A. S. G., Bland-Hawthorn, J., et al. 2013, MNRAS, 430, 2622

Driver, S. P., Andrews, S. K., Davies, L. J., et al. 2016a, ApJ, 827, 108

Driver, S. P., Wright, A. H., Andrews, S. K., et al. 2016b, MNRAS, 455, 3911
Dunne, L., Eales, S., Edmunds, M., et al. 2000, MNRAS, 315, 115 Duras, F., Bongiorno, A., Piconcelli, E., et al. 2017, A\&A, 604, A67 Eales, S., Dunne, L., Clements, D., et al. 2010, PASP, 122, 499 Eales, S., de Vis, P., Smith, M. W. L., et al. 2017, MNRAS, 465, 3125 Edmunds, M. G. 2001, MNRAS, 328, 223

Eisenstein, D. J., Weinberg, D. H., Agol, E., et al. 2011, AJ, 142, 72 Engelbracht, C. W., Hunt, L. K., Skibba, R. A., et al. 2010, A\&A, 518, L56 Ferland, G. J., Korista, K. T., Verner, D. A., et al. 1998, PASP, 110, 761 Fioc, M., \& Rocca-Volmerange, B. 1997, A\&A, 326, 950 Franceschini, A., \& Rodighiero, G. 2017, A\&A, 603, A34 Franceschini, A., Andreani, P., \& Danese, L. 1998, MNRAS, 296, 709 Franceschini, A., Aussel, H., Cesarsky, C. J., Elbaz, D., \& Fadda, D. 2001, A\&A, 378,1

Franceschini, A., Rodighiero, G., \& Vaccari, M. 2008, A\&A, 487, 837 Franceschini, A., Rodighiero, G., Vaccari, M., et al. 2010, A\&A, 517, A74 Galliano, F., Galametz, M., \& Jones, A. P. 2018, ARA\&A, 56, 673 Grand, R. J. J., Gómez, F. A., Marinacci, F., et al. 2017, MNRAS, 467, 179 Griffin, M. J., Abergel, A., Abreu, A., et al. 2010, A\&A, 518, L3 Hauser, M. G., Arendt, R. G., Kelsall, T., et al. 1998, ApJ, 508, 25 Hayward, C. C., \& Smith, D. J. B. 2015, MNRAS, 446, 1512 James, A., Dunne, L., Eales, S., \& Edmunds, M. G. 2002, MNRAS, 335, 753 Jones, A. P., Köhler, M., Ysard, N., Bocchio, M., \& Verstraete, L. 2017, A\&A, 602, A46

Kelvin, L. S., Driver, S. P., Robotham, A. S. G., et al. 2014, MNRAS, 439, 1245 Kennicutt, R. C., Calzetti, D., Aniano, G., et al. 2011, PASP, 123, 1347 Kessler, M. F., Steinz, J. A., Anderegg, M. E., et al. 1996, A\&A, 315, L27 Lutz, D. 2014, ARA\&A, 52, 373

Magnelli, B., Lutz, D., Saintonge, A., et al. 2014, A\&A, 561, A86

Makarov, D., Prugniel, P., Terekhova, N., Courtois, H., \& Vauglin, I. 2014, A\&A, 570, A13

Morrissey, P., Conrow, T., Barlow, T. A., et al. 2007, ApJS, 173, 682

Mosenkov, A. V., Allaert, F., Baes, M., et al. 2016, A\&A, 592, A71

Mosenkov, A. V., Allaert, F., Baes, M., et al. 2018, A\&A, 616, A120 Mosenkov, A. V., Baes, M., Bianchi, S., et al. 2019, A\&A, in press, DOI: $10.1051 / 0004-6361 / 201833932$

Neugebauer, G., Habing, H. J., van Duinen, R., et al. 1984, ApJ, 278, L1

Noll, S., Burgarella, D., Giovannoli, E., et al. 2009, A\&A, 507, 1793

Pilbratt, G. L., Riedinger, J. R., Passvogel, T., et al. 2010, A\&A, 518, L1

Poglitsch, A., Waelkens, C., Geis, N., et al. 2010, A\&A, 518, L2

Polletta, M., Tajer, M., Maraschi, L., et al. 2007, ApJ, 663, 81

Popescu, C. C., \& Tuffs, R. J. 2002, MNRAS, 335, L41

Popescu, C. C., Misiriotis, A., Kylafis, N. D., Tuffs, R. J., \& Fischera, J. 2000, A\&A, 362, 138

Puget, J.-L., Abergel, A., Bernard, J.-P., et al. 1996, A\&A, 308, L5

Rémy-Ruyer, A., Madden, S. C., Galliano, F., et al. 2015, A\&A, 582, A121

Riedinger, J. 2009, ESA Bull., 139, 14

Roehlly, Y., Burgarella, D., Buat, V., et al. 2014, ASP Conf. Ser., 485, 347

Rowlands, K., Gomez, H. L., Dunne, L., et al. 2014, MNRAS, 441, 1040

Saftly, W., Baes, M., De Geyter, G., et al. 2015, A\&A, 576, A31

Salpeter, E. E. 1955, ApJ, 121, 161

Schaye, J., Crain, R. A., Bower, R. G., et al. 2015, MNRAS, 446, 521

Schneider, R., Bianchi, S., Valiante, R., Risaliti, G., \& Salvadori, S. 2015, A\&A, 579, A60

Sérsic, J. L. 1963, Bol. Asoc. Argent. Astron. Plata Argent., 6, 41

Sérsic, J. L. 1968, Atlas de Galaxias Australes (Córdoba, Argentina: Observatorio Astronómico)

Silva, L., Granato, G. L., Bressan, A., \& Danese, L. 1998, ApJ, 509, 103

Skibba, R. A., Engelbracht, C. W., Dale, D., et al. 2011, ApJ, 738, 89

Skrutskie, M. F., Cutri, R. M., Stiening, R., et al. 2006, AJ, 131, 1163

Smith, D. J. B., Dunne, L., da Cunha, E., et al. 2012a, MNRAS, 427, 703

Smith, M. W. L., Gomez, H. L., Eales, S. A., et al. 2012b, ApJ, 748, 123

Soifer, B. T., \& Neugebauer, G. 1991, AJ, 101, 354

Soifer, B. T., Boehmer, L., Neugebauer, G., \& Sanders, D. B. 1989, AJ, 98, 766

Strateva, I., Ivezić, Ž., Knapp, G. R., et al. 2001, AJ, 122, 1861

Symeonidis, M., Vaccari, M., Berta, S., et al. 2013, MNRAS, 431, 2317

Trayford, J. W., Camps, P., Theuns, T., et al. 2017, MNRAS, 470, 771

Vagshette, N. D., Pandge, M. B., Pandey, S. K., \& Patil, M. K. 2012, New Astron., 17, 524

Varenius, E., Conway, J. E., Martí-Vidal, I., et al. 2014, A\&A, 566, A15

Viaene, S., Baes, M., Bendo, G., et al. 2016, A\&A, 586, A13

Viaene, S., Baes, M., Tamm, A., et al. 2017, A\&A, 599, A64

Wang, B., \& Heckman, T. M. 1996, ApJ, 457, 645

Wang, L., Dutton, A. A., Stinson, G. S., et al. 2015, MNRAS, 454, 83

Wright, E. L., Eisenhardt, P. R. M., Mainzer, A. K., et al. 2010, AJ, 140, 1868

Xilouris, E. M., Byun, Y. I., Kylafis, N. D., Paleologou, E. V., \& Papamastorakis, J. 1999, A\&A, 344, 868

Xu, C., \& Buat, V. 1995, A\&A, 293, L65

Young, L. M., Serra, P., Krajnović, D., \& Duc, P.-A. 2018, MNRAS, 477, 2741 


\section{Appendix A: Comparison between different $f_{\text {abs }}$ estimates}

As described in Sect. 2.2, in this work we derived $f_{\text {abs }}$ from the Bayesian estimates for $L_{\text {bolo }}$ and $L_{\text {dust }}$ given by CIGALE. In CIGALE, $L_{\text {bolo }}$ and $L_{\text {dust }}$ are computed for each model by integrating the SED over the entire wavelength grid, though we set as a lower limit $\lambda=0.0912 \mu \mathrm{m}$ (to exclude ionising radiation); also, for the modules we used, emission at $\lambda>1000 \mu \mathrm{m}$ constitutes a negligible contribution to the luminosities. The Bayesian estimates are derived from all the models, while best-fit luminosities are derived from the model which minimise the $\chi^{2}$.

At earlier stages we have experimented a variety of other methods: a direct interpolation through the photometric datapoints; the use of CIGALE's best-fit luminosities; an independent estimate of the parameters using the MAGPHYS fitting code. We report here briefly on the main differences between these approaches. The three panels of Fig. A.1 show the comparison between the reference estimate of $f_{\text {abs }}$ (on the y-axis) and each of alternative ones (on the $\mathrm{x}$-axis), together with a linear fit to them.

\section{A.1. Direct integration}

When the wavelength range dominated by non-ionising stellar and dust radiation $(0.0912<\lambda / \mu \mathrm{m}<1000)$ is covered uniformly by a good number of good-quality photometric datapoints, it is possible to construct a piecewise representation of the SED. $L_{\text {bolo }}$ can be derived by integrating this SED over the whole wavelength range. For the estimate of $L_{\text {dust }}$ one can assume as a lower integration limit a NIR wavelength where the dust emission starts to dominate over the stellar emission, or include a correction to take into account the (declining) stellar contribution at longer wavelengths. Here we have used the former method and derive $L_{\text {dust }}$ by integrating the SED from the WISE $3.4 \mu \mathrm{m}$ band. The latter method was used in the analogous estimates by Skibba et al. (2011) on the KINGFISH sample. We have used a sub-sample of DustPedia galaxies for which photometry is available at $>2 \sigma$ level in all GALEX, Sloan Digital Sky Survey (SDSS; Eisenstein et al. 2011), 2 Micron AllSky Survey (2MASS; Skrutskie et al. 2006), WISE, and SPIRE wavebands, and in at least two Herschel-Photodetector Array Camera and Spectrometer (PACS; Poglitsch et al. 2010) wavebands. Only 155 galaxies have at least this coverage. We extrapolated the SED to $912 \AA$ by assuming $\lambda \times F_{\lambda}=$ constant for $0.0912<\lambda / \mu \mathrm{m}<0.15$, and to $1000 \mu \mathrm{m}$, assuming a modified black-body of $T=20 \mathrm{~K}$ and an absorption cross section $\kappa_{\lambda} \propto \lambda^{-\beta}$ with $\beta=1.6$ (typical values for the diffuse cirrus in the MW; see, e.g. Bianchi et al. 2017). These extrapolations, and in particular the extension down to the Lyman break, change the estimate of $f_{\text {abs }}$ by less than $1 \%$. Of similar order are the effects on the choice of the limit wavelength in the estimate of $L_{\text {dust }}$ : if instead of a sharp cut the stellar contribution at $\lambda \geq 3.4 \mu \mathrm{m}$ is assumed to follow a Rayleigh-Jeans spectrum, $f_{\text {abs }}$ changes by about $1.0 \%$.

The comparison between this method and the reference method is shown in the left panel of Fig. A.1. The fit yields $f_{\text {abs }}($ Bayes $)=1.06 \times f_{\text {abs }}$ (integration) $-3.5 \%$. For $f_{\text {abs }}>20 \%$ (integration) the agreement is good and within the average CIGALE error provided by the Bayesian analysis, $3 \%$ for the same sample of galaxies. For $f_{\mathrm{abs}}<20 \%$, the direct integration of the piecewise SED leads to higher values of $f_{\text {abs }}$. This is mostly due to the straight-line nature of the piecewise SED over the MIR features, and over the $25 \lesssim \lambda / \mu$ m $\lesssim 60$ range, where no data is available. An example of this behaviour is shown in
Fig. 1 for NGC 3254. The effect can bias the estimate of $f_{\text {abs }}$ high, especially when the FIR peak is low.

\section{A.2. CIGALE best fits}

In a second test, we used CIGALE best-fit luminosities to derive $f_{\text {abs }}$. The comparison of the Bayesian and best-fit estimates is shown in the central panel of Fig. A.1. In general, there is a good agreement between the two estimates, with $f_{\text {abs }}$ (Bayes) $=$ $1.0 \times f_{\text {abs }}$ (best) $-0.3 \%$. For $f_{\text {abs }}<10 \%$, the Bayesian estimate is slightly lower than the best-fit one, but still consistent with the average Bayesian error estimate on the same sample, $1 \%$. In a few instances, though, the discrepancy is larger: they correspond, however, to objects where most of the (dust emission) flux densities are consistent with zero within a few $\sigma$, and the fit is poorly constrained. In particular for these cases, the Bayesianlike approach provides a more conservative and robust estimate, which is less prone to the degeneracies in the SED fit.

\section{A.3. MAGPHYS}

For late-type galaxies only, we also modelled the SED using the MAGPHYS ${ }^{8}$ code of da Cunha et al. (2008). MAGPHYS uses libraries of physically justified optical and IR models. The un-attenuated stellar SED is computed by assuming a Chabrier (2003) initial mass function and using the 2007 version of the Bruzual \& Charlot (2003) stellar population synthesis models (see Bruzual 2007). The star-formation history (SFH) is given by a continuous exponentially declining SFH, with random bursts of star formation superimposed. The attenuation by dust is described by the prescription of Charlot \& Fall (2000) and includes attenuation in birth clouds (i.e. molecular clouds where stars form) and in the ambient (i.e. diffuse) interstellar medium (ISM). The dust emission is made up of four different components: polycyclic aromatic hydrocarbons (PAHs), small stochastically heated grains that produce mid-infrared continuum emission and warm and cold dust in thermal equilibrium with the radiation field. The warm and cold components are described using modified black-body spectra and absorption cross section with spectral indices $\beta=1.5$ and 2.0 respectively, normalised to $\kappa_{850 \mu \mathrm{m}}=0.077 \mathrm{~m}^{2} \mathrm{~kg}^{-1}$ (Dunne et al. 2000; James et al. 2002).

Within MAGPHYS, 50000 optical models and 50000 IR models are combined together maintaining energy balance, that is, the energy absorbed between the attenuated and unattenuated SEDs in the optical model, is equal to the energy emitted in the IR model. The large number of templates obtained in this way is compared to the multi-wavelength photometry and a goodnessof-fit $\chi^{2}$ is calculated. By running over each template, probability density functions (PDF) are made for the parameters used to build each template by weighting the value of that parameter by the probability $\mathrm{e}^{-\chi^{2}}$ corresponding to that template. The median values of these PDF produce reliable estimates for each parameter (Hayward \& Smith 2015) and the corresponding uncertainties are the 16th and 84th percentiles of the PDF. As in Viaene et al. (2016), we used the PDF-derived values for $L_{\text {dust }}$ (directly provided by code, after integrating the modelled SED over the code's grid), while we estimated $L_{\text {bolo }}$ by integrating the best-fit SED in $0.0912<\lambda / \mu \mathrm{m}<1000$. The uncertainty in $f_{\text {abs }}$ is assumed to be entirely due to the uncertainty in $L_{\text {dust }}$, as is also verified in CIGALE fits.

8 Publicly available at: www .iap. fr/magphys 


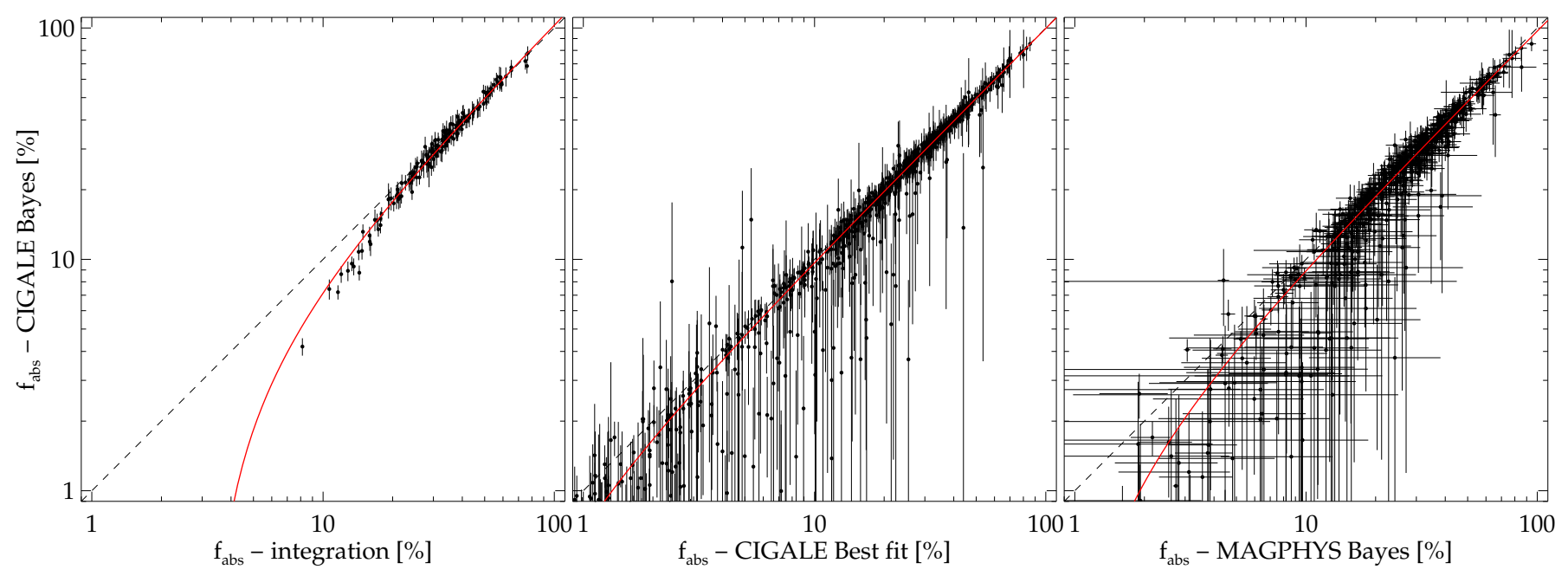

Fig. A.1. Comparison between the $f_{\text {abs }}$ estimated used in the main text and alternative estimates for the quantity. The dashed line shown the 1:1 relation. The red line is a linear fit between the estimates.

We made adaptations to MAGPHYS in order to be able to consistently compare with CIGALE. First, we allow the use of the (few) negative fluxes, yet consistent with zero at the $2 \sigma$ level, present in the database. These negative fluxes still provide constrains on the data and can easily be incorporated in the $\chi^{2}$ calculation. Second, we extended the MAGPHYS infrared library to the temperature range 10-30 K, which encompasses better the properties of galaxies in our sample (see also Viaene et al. 2016; De Vis et al. 2017a). Third, CIGALE adds an extra source of error ( $10 \%$ of the flux) to each photometric point to take into account uncertainties in the models (Boquien, priv. comm.). We add the same uncertainty to our photometry before fitting the SEDs with MAGPHYS.

The right panel of Fig. A.1 shows the comparison between the CIGALE and MAGPHYS estimates (in this panel, we also plot on the $\mathrm{x}$-axis the uncertainties derived from MAGPHYS). Again, there is a good correspondence between the estimates, with $f_{\text {abs }}($ CIGALE $)=0.98 \times f_{\text {abs }}($ MAGPHYS $)-0.9 \%$. The average difference and scatter between CIGALE and MAGPHYS is $\Delta f_{\text {abs }}=-2.2 \pm 4.3 \%$ for the whole late-type sample. The scatter is compatible with the average errors on $f_{\text {abs }}$, which is $3.3 \%$ for both methods, leading to an average error on the difference of $4.7 \%$. Given the diversity of the physical recipes and of the methods used by the codes for fitting the SED, and for deriving PDF-based estimates and uncertainties, it is difficult to understand the reason for the slightly higher MAGPHYS values for $f_{\text {abs }}$. We note, however, that MAGPHYS has a tendency to produce higher $f_{\text {abs }}$ values when the SED coverage in the MIR region between 24 and $100 \mu \mathrm{m}$ is insufficient: a large, unconstrained, component from the warm dust template might be present which instead is not seen in the physicallybased emission templated of CIGALE. An example of this unconstrained bump is shown by the MAGPHYS fit of UGC 5692 in Fig. 1.

\section{A.4. Differences in the results}

Despite the differences in the various method we experimented, they affect little the main conclusions of this work. For examples, for LTGs $\left\langle f_{\text {abs }}\right\rangle=24.9 \pm 0.7 \%$ using the reference model, $25.6 \pm$ $0.7 \%$ for CIGALE best-fit, and $27.2 \pm 0.7$ for MAGPHYS; for galaxies later than $\mathrm{Sb}, f_{\text {abs }}$ vs. $L_{\text {bolo }}$ has a Kendall's correlation measure $\tau_{\mathrm{K}}=0.54$ for CIGALE's Bayesian estimate, 0.52 for
CIGALE best-fit, and 0.5 for MAGPHYS. Finally, there is little difference (at least in the determination of $f_{\text {abs }}$ ) if the different dust properties of the dl14 dust emission module (see Sect. 2.2) are used: for LTGs, it is $\left\langle f_{\text {abs }}\right\rangle=25.1 \pm 0.7 \%$; for types later than Sb, $f_{\text {abs }}$ vs. $L_{\text {bolo }}$ has $\tau_{\mathrm{K}}=0.54$.

\section{Appendix B: $f_{\text {abs }}$ from models of edge-on galaxies}

We show here that typical RT models of edge-on galaxies with prominent dust-extinction lanes imply a sizeable dependence of $f_{\text {abs }}$ on inclination. De Geyter et al. (2014) fitted the opti$\mathrm{cal} / \mathrm{NIR}$ images of a sample of 12 edge-on galaxies with diffuse, homogeneous, geometrical components, using an automated routine, FitSKIRT (De Geyter et al. 2013) based on the code SKIRT (Baes \& Camps 2015). In order to study the inclination effects on our results, we simulated a galaxy with the average properties of the sample fitted by De Geyter et al. (2014, see their Table 4), composed of an exponential disk and a bulge for stars, and an exponential disk for dust. We used the code SKIRT, its built-in template for an Sc galaxy spectrum (from Fioc \& Rocca-Volmerange 1997) and the optical properties of the THEMIS dust model.

The models were produced for several galactic inclinations, and $f_{\text {abs }}$ estimated from the mock SEDs in a way analogous to that adopted for real observations, integrating the output SEDs over the range $0.0912<\lambda / \mu \mathrm{m}<1000$ and removing the contribution of un-attenuated starlight in the NIR when deriving $L_{\text {dust }}$ (see Sect. A). The results are shown in Fig. B.1 (blue symbols) and compared to the true $f_{\text {abs }}$, meaning the fraction of stellar radiation that is absorbed within the model globally, directly derived from the RT modelling. As expected, stellar emission is not isotropic: not only it is reduced at higher inclinations, because of dust extinction; it is also enhanced at face-on inclinations, because UV-optical radiation originally travelling along the galactic plane is scattered by dust in directions close to the galaxy axis. The dust emission, instead, is almost isotropic. As a consequence, $f_{\text {abs }} \approx 12 \%$ for the face-on case, smaller that true value obtained from the RT, $14 \%$. It increases to $18 \%$ for the edgeon case. It is worth noting that the estimated $L_{\mathrm{bolo}}$ decreases by $\approx 32 \%$ between the two inclination extremes.

It is well known that the average fit to edge-on galaxies is not able to reproduce the amount of radiation observed in the FIR: a factor of approximately three higher opacity (or, for 
S. Bianchi et al.: Fraction of bolometric luminosity absorbed by dust in DustPedia galaxies

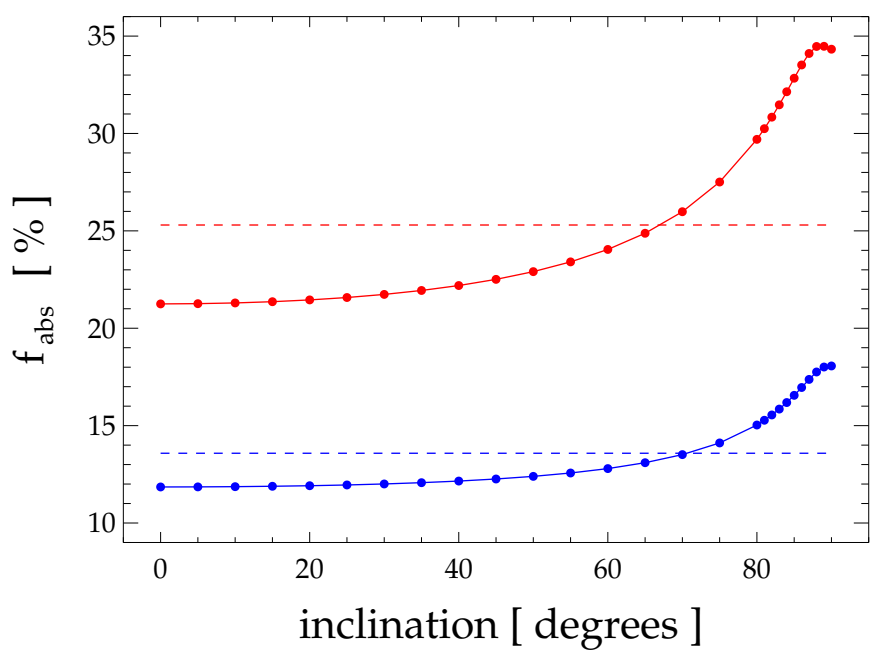

Fig. B.1. $f_{\text {abs }}$ vs. inclination estimated from the SEDs of the average edge-on galaxy model of De Geyter et al. (2014, blue lines and symbols), and from the model with $3 \times$ the dust mass (red lines and symbols). In both cases, dashed lines refers to the true $f_{\text {abs }}$ derived from the RT energy budget. the same dust properties, an approximate threefold increase in the dust mass) is required by the energy conservation (see, e.g. Mosenkov et al. 2018, and the other references in their introduction). If we increase the dust mass of the average model by a factor 3 (red symbols in Fig. B.1), the true $f_{\mathrm{abs}}$ rises to $25 \%$, while the estimated $f_{\text {abs }}$ ranges from 21 to $34 \%$ going from the face-on to the edge-on case; $L_{\text {bolo }}$ decreases by $38 \%$. In this second model also dust emission shows a (moderate) anisotropy, due to the self-absorption of radiation emitted at shorter wavelength by non-thermal, stochastic, processes. Thus, RT models commonly fitted to edge-on galaxies suggest differences between the edge-on and face-on $f_{\text {abs }}$ of $\approx 5-15 \%$. 